\title{
Sobolev regular solutions for the incompressible Navier-Stokes equations in higher dimensions: asymptotics and representation formulae
}

\author{
Weiping Yan ${ }^{1}$ - Vicențiu D. Rădulescu ${ }^{2,3}$
}

Received: 10 June 2020 / Accepted: 4 July 2020 / Published online: 25 July 2020

(c) The Author(s) 2020

\begin{abstract}
In this paper, we consider the steady incompressible Navier-Stokes equations in a smooth bounded domain $\Omega \subset \mathbb{R}^{n}$ with the dimension $n \geq 3$. We first establish asymptotic expansion formulae of Sobolev regular finite energy solutions in $\Omega$. In the second part of this paper, explicit representation formulae of Sobolev regular solutions are showed in the regular polyhedron $\Omega:=[0, T]^{n}$.
\end{abstract}

Keywords Navier-Stokes equations · Sobolev regular solution · Nash-Moser iteration · Calderon-Zygmund theory

Mathematics Subject Classification 35Q31 $\cdot 35 \mathrm{~A} 01 \cdot 76 \mathrm{D} 05$

\section{Introduction and main results}

In this paper, we consider the steady incompressible Navier-Stokes equations:

$$
\left\{\begin{array}{l}
-v \Delta U+U \cdot \nabla U+\nabla P=f, \\
\nabla \cdot U=0,
\end{array}\right.
$$

This paper is dedicated to the memory of Professor Ciprian Foiaş (1933-2020), a Master of NavierStokes equations.

Vicenţiu D. Rădulescu

radulescu@inf.ucv.ro

Weiping Yan

yanwp@xmu.edu.cn

1 School of Mathematics, Xiamen University, Xiamen 361000, People's Republic of China

2 Faculty of Applied Mathematics, AGH University of Science and Technology, Al. Mickiewicza 30, 30-059 Kraków, Poland

3 Department of Mathematics, University of Craiova, Street A.I. Cuza No. 13, 200585 Craiova, Romania 
where $x \in \Omega$, and $\Omega \subset \mathbb{R}^{n}$ is a smooth bounded domain, $U: \mathbb{R}^{+} \times \Omega \rightarrow \mathbb{R}^{n}$ is the fluid velocity, and it is of the form $U(x)=\left(U_{1}(x), U_{2}(x), \ldots, U_{n}(x)\right), P(x): \mathbb{R}^{+} \times \Omega \rightarrow \mathbb{R}$ stands for the pressure in the fluid, and the constant $v$ is the viscosity. We denote by $f:=\left(f_{1}, f_{2}, \ldots, f_{n}\right)$ an external force. The divergence free condition in second equations of (1.1) guarantees the incompressibility of the fluid.

We supplement the steady incompressible Navier-Stokes equations (1.1) with the Dirichlet boundary condition

$$
\left.U(x)\right|_{x \in \partial \Omega}=0
$$

and the pressure takes the form

$$
\Delta P(x)=-\sum_{i, j=1}^{n} \frac{\partial U_{i}}{\partial x_{j}} \frac{\partial U_{j}}{\partial x_{i}} .
$$

In particular, when the external force $f=0$ in equations (1.1), then our problem reduces to the steady incompressible Navier-Stokes equations

$$
\left\{\begin{array}{l}
U_{t}-v \Delta U+U \cdot \nabla U+\nabla P=0, \\
\nabla \cdot U=0 .
\end{array}\right.
$$

The question of whether a solution of the 3D incompressible Navier-Stokes equations can develop a finite time singularity from smooth initial data with finite energy is one of the Millennium Prize problems, see[8]. In 1934, Leray[22] showed that the 3D incompressible Navier-Stokes equations (1.1) admit global-forward-in-time weak solutions of the initial value problem. Caffarelli, Kohn and Nirenberg[6] established a $\varepsilon$-regularity criterion for equations (1.1). After that, Lin[23] gave a new and simpler proof for the result of Caffarelli, Kohn and Nirenberg. Koch and Tataru[21] proved the global well-posedness for the equations (1.1) in a space of arbitrary dimension with small initial data in $B M O^{-1}$ space. Recently, Buckmaster and Vicol[5] proved that the Leray weak solutions of the $3 D$ Navier-Stokes equations are not unique in the class of weak solutions with finite kinetic energy. We refer the readers to[2-4, 9, 19, 28, 34-34] for more related results on this equations.

Much attention attracted the existence and the regularity properties of stationary solutions for the incompressible Navier-Stokes equations. These solutions depend on the force $f$ and the domain $\Omega$. Gerhardt[15] proved that the steady four-dimensional problem admits a solution in $W^{2, p}$ by assumption the force $f \in L^{p}$. Frehse and Ruzicka[11] and Struwe[31] got the existence and regularity of the solutions in the fivedimensional case, respectively. Frehse and Ruzicka[10] also obtained the existence of regular solutions in a bounded domain of six-dimension. The fifteen dimensional torus case was given in[12]. Maz'ya and Rossmann[25] showed the existence of weak solutions in the three-dimensional case for a polyhedral domain. Kim[20] considered the existence of very weak solutions in a bounded domain of dimension $d=2,3,4$. Farwig and Sohr[7] proved the existence, uniqueness and very low regularity of solutions to the inhomogeneous Navier-Stokes equations with special external force in a bounded domain of $d \geq 3$ dimension. Recently, Hou and Pei[18] got the existence of weak solutions in a bounded connected polygon or polyhedron of two or three dimension. Luo[24] obtained the non-uniqueness of weak solutions for this kind of problems in the case of the $d$-torus with $d \geq 4$. We cannot list all the contributions to this field, but we refer the readers to[12-14] and the references therein. 
As pointed out in[24], the question of uniqueness of regular solutions to the steady incompressible Navier-Stokes equations (1.1) remains mostly an open problem. The nonuniqueness of the weak solution has been given in[24]. In this paper, under the assumption of the external force $f$ being small and $f \not \equiv 0$, we give asymptotic expansion formulae of Sobolev regular solution with finite energy for the steady incompressible Navier-Stokes equations (1.1) in a smooth bounded domain $\Omega$ of dimension $n \geq 3$. Next, we give explicit representation formulae of the Sobolev regular solution in a special domain, namely if $\Omega:=([0, T])^{n}$.

We now state the main result in this paper.

Theorem 1.1 Let the viscous constant $v \geq 1$ and $s \geq 1$. Asssume that the external force $f \in H^{s}(\Omega)$ with $\|f\|_{H^{s}(\Omega)} \lesssim \varepsilon$ and $f \not \equiv 0$. Then the steady incompressible Navier-Stokes equations (1.1) with the Dirichlet boundary conditions (1.2) admit a Sobolev regular solution with finite energy $U \in H^{s}(\Omega)$. Here, the pressure is given by (1.3).

Moreover, there exists a small constant $0<\varepsilon \ll 1$ such that

$$
\begin{aligned}
\|U(x)\|_{H^{s}(\Omega)} & \lesssim \varepsilon, \\
\|P(x)\|_{H^{s}(\Omega)} & \lesssim \varepsilon,
\end{aligned}
$$

for any $x \in \Omega \subset \mathbb{R}^{n}(n \geq 3)$.

Remark 1.1 The main feature of Theorem 1.1 is that it gives explicit representation formulae as follows

$$
U^{(\infty)}(x)=U^{(0)}(x)+\sum_{m=1}^{\infty} \mathbf{h}^{(m)}(x)=U^{(0)}(x)+\mathcal{O}\left(\varepsilon^{2}\right),
$$

where the function $U^{(0)}(x)$ satisfies the assumption

$$
\left\{\begin{array}{l}
\nabla \cdot U^{(0)}(t, x)=0, \\
\left\|U^{(0)}\right\|_{H^{s}(\Omega)} \lesssim \varepsilon, \\
\left.U^{(0)}(x)\right|_{x \in \partial \Omega}=0 .
\end{array}\right.
$$

and

$$
\sum_{k=0}^{s}\left\|\partial_{x_{i}}^{k} U_{j}^{(0)}(x)\right\|_{L^{\infty}} \lesssim \varepsilon_{0}, \quad \forall i, j=1,2, \ldots, n,
$$

and $\mathbf{h}^{(m)}(x)(m=1,2,3, \ldots)$ is obtained by solving the linearized problem with the Dirichlet boundary condition in Sobolev space $H^{s}(\Omega)$ with $s>1$

$$
\left\{\begin{array}{l}
\mathcal{L}\left[U^{m-1}\right] \mathbf{h}^{(m)}=E^{(m-1)}(x), \\
\nabla \cdot \mathbf{h}^{(m)}=0 \\
\left.\mathbf{h}^{(m)}(x)\right|_{x \in \partial \Omega}=0
\end{array}\right.
$$

and $E^{(m-1)}(x)$ denotes the error term, while the linear operator $\mathcal{L}\left[U^{m-1}\right] \mathbf{h}^{(m)}$ is defined in (2.7). The index $s$ of the Sobolev regularity depends on the higher derivative estimate of solution for the linearized equations. From (1.4), we remark that the solution depends 
strongly on the initial approximation function $U^{(0)}(x)$. Our proof is based on the NashMoser iteration scheme, by using some ideas developed in [37, 38]. For the general NashMoser implicit function theorem, we refer to the seminal papers of Nash [27], Moser [26] and Hörmander [17], and to Rabinowitz [29] for a singular perturbation problem of elliptic equations by using the Nash-Moser implicit function theorem.

In particular, if we consider the domain $\Omega:=([0, T])^{n}$ (a regular polyhedron) with the finite constant $T>0$, then we have the following explicit representation formulae.

Corollary 1.1 Let the integers $p>1$ and $q>2$, and the parameter $0<\varepsilon \ll 1$. Then the steady incompressible Navier-Stokes equations (1.1) admit an explicit expansion of the Sobolev regular solution with finite energy as follows

$$
U^{*}(x)=\left(U_{1}^{(0)}(x), U_{2}^{(0)}(x), \ldots, U_{n}^{(0)}(x)\right)+\mathcal{R}(x),
$$

for all $x=\left(x_{1}, x_{2}, \ldots, x_{n}\right) \in \Omega:=([0, T])^{n}$, and

$$
\left\{\begin{aligned}
U_{1}^{(0)}(x) & :=\varepsilon x_{1}^{q}\left(x_{1}-T\right)^{q} g\left(x_{2}\right) g\left(x_{3}\right) e^{-r\left(x_{1}, x_{2}, x_{3}\right)}, \\
U_{2}^{(0)}(x) & :=\varepsilon x_{2}^{q}\left(x_{2}-T\right)^{q} g\left(x_{1}\right) g\left(x_{3}\right) e^{r\left(x_{1}, x_{2}, x_{3}\right)} \\
U_{3}^{(0)}(x) & :=-2 \varepsilon x_{3}^{q}\left(x_{3}-T\right)^{q} g\left(x_{1}\right) g\left(x_{2}\right) e^{-r\left(x_{1}, x_{2}, x_{3}\right)} \\
U_{4}^{(0)}(x) & :=0, \\
& \cdots, \\
U_{n}^{(0)}(x) & :=0
\end{aligned}\right.
$$

where

$$
\begin{aligned}
g(y) & :=y^{q-1}(y-T)^{q-1}(2 y-T)\left(q-2(p+1) y^{2(p+1)}(y-T)^{2(p+1)}\right), \\
r\left(x_{1}, x_{2}, x_{3}\right) & :=\sum_{k=1}^{3} x_{k}^{2(p+1)}\left(x_{k}-T\right)^{2(p+1)},
\end{aligned}
$$

and the remainder term $\mathcal{R}(x) \in H^{s}(\Omega)$ satisfies

$$
\begin{aligned}
& \nabla \cdot \mathcal{R}(x)=0, \\
& \left.\mathcal{R}(x)\right|_{x \in \partial \Omega}=0, \\
& \|\mathcal{R}(x)\|_{H^{s}(\Omega)} \sim \mathcal{O}\left(\varepsilon^{2}\right) .
\end{aligned}
$$

Moreover, the pressure is determined by

$$
\Delta P^{*}(x)=-\sum_{i, j=1}^{3} \frac{\partial U_{i}^{*}}{\partial x_{j}} \frac{\partial U_{j}^{*}}{\partial x_{i}} .
$$

Notations Throughout this paper, we assume that $\Omega \subset \mathbb{R}^{n}$ with $n \geq 3$ and we denote the usual norms of $\mathbb{L}^{2}(\Omega)$ and $\mathbb{U}^{s}(\Omega)$ by $\|\cdot\|_{\mathbb{L}^{2}}$ and $\|\cdot\|_{\mathbb{A}^{s}}$, respectively. The norm of the Sobolev space $H^{s}(\Omega):=\left(\mathbb{M}^{s}(\Omega)\right)^{n}$ is denoted by $\|\cdot\|_{H^{s}}$. The symbol $a \lesssim b$ means that there exists a positive constant $C$ such that $a \leq C b$. We denote by $\left(x_{1}, x_{2}, x_{3}, \ldots, x_{n}\right)^{T}$ the column vector in $\mathbb{R}^{n}$. The letter $C$ with subscripts to denote dependencies stands for a positive constant that might change its value at each occurrence. 
The paper is organized as follows. In Sect. 2, we first give a class of initial approximation functions, then the Carleman-type estimate of solution for the linearized equations about the initial approximation functions is shown. Next, we prove the existence of the Sobolev regular solution for the linearized equations. In Sect. 3, we establish the general approximation step for the construction of the Nash-Moser iteration scheme. In the final section of this paper, we show how to construct a small Sobolev regular solution for the incompressible steady Navier-Stokes equations (1.1) by the proof of convergence for the Nash-Moser iteration scheme.

\section{The first approximation step}

We introduce a family of smooth operators possessing the following properties.

Lemma $2.1[1,17]$ There is a family $\left\{\Pi_{\theta}\right\}_{\theta \geq 1}$ of smoothing operators in the space $H^{s}(\Omega)$ acting on the class of functions such that

$$
\begin{aligned}
& \left\|\Pi_{\theta} U\right\|_{H^{s_{1}(\Omega)}} \leq C \theta^{\left(s_{1}-s_{2}\right)_{+}}\|U\|_{H^{k_{2}(\Omega)}}, \quad \forall s_{1}, s_{2} \geq 0, \\
& \left\|\Pi_{\theta} U-U\right\|_{H^{s_{1}(\Omega)}} \leq C \theta^{s_{1}-s_{2}}\|U\|_{\left.H^{s_{2}(\Omega)}\right)}, 0 \leq s_{1} \leq s_{2}, \\
& \left\|\frac{d}{d \theta} \Pi_{\theta} U\right\|_{H^{s_{1}(\Omega)}} \leq C \theta^{\left(s_{1}-s_{2}\right)_{+}-1}\|U\|_{H^{s_{2}(\Omega)}}, \quad \forall s_{1}, s_{2} \geq 0,
\end{aligned}
$$

where $C$ is a positive constant and $\left(s_{1}-s_{2}\right)_{+}:=\max \left(0, s_{1}-s_{2}\right)$.

In our iteration scheme, we set

$$
\theta=N_{m}=N_{0}^{m}, \quad \forall m=0,1,2, \ldots,
$$

where $N_{0}$ is a fixed positive constant, then by (2.1), it follows that

$$
\left\|\Pi_{N_{m}} U\right\|_{H^{s_{1}}(\Omega)} \lesssim N_{m}^{s_{1}-s_{2}}\|U\|_{H^{s_{2}}(\Omega)}, \quad \forall s_{1} \geq s_{2} .
$$

We consider the approximation problem of the steady incompressible Navier-Stokes equations (1.1) as follows

$$
\mathcal{J}(U):=-v \Delta U+\Pi_{N_{m}}(U \cdot \nabla U+\nabla P)-f
$$

with the Dirichlet boundary condition (1.2) and the incompressible condition

$$
\nabla \cdot U=0 .
$$

\subsection{The initial approximation function}

Let $s \geq 1$ be a fixed finite constant and $0<\varepsilon_{0}<\varepsilon^{2} \ll 1$. For any $x \in \Omega$, we choose the initial approximation functions

$$
U^{(0)}(x)=\left(U_{1}^{(0)}(x), U_{2}^{(0)}(x), \ldots, U_{n}^{(0)}(x)\right)^{T} \in H^{s}(\Omega) .
$$

Meanwhile, we require 


$$
\left\{\begin{array}{l}
\nabla \cdot U^{(0)}(x)=0, \\
\left\|U^{(0)}\right\|_{H^{s}} \lesssim \varepsilon_{0}, \\
\left.U^{(0)}(x)\right|_{x \in \partial \Omega}=0 .
\end{array}\right.
$$

Moreover, for any fixed constant $s \geq 1$ and $x \in \Omega$ and $i, j=1,2, \ldots, n$, we also need the condition

$$
\sum_{k=0}^{s}\left\|\partial_{x_{i}}^{k} U_{j}^{(0)}(x)\right\|_{L^{\infty}} \lesssim \varepsilon_{0}, \quad \forall i, j=1,2, \ldots, n,
$$

and the initial error term

$$
\left\|E^{(0)}\right\|_{H^{s}} \lesssim \varepsilon_{0}
$$

where $E^{(0)}$ denotes the error term taking the form

$$
E^{(0)}:=\mathcal{J}\left(U^{(0)}\right),
$$

with

$$
\Delta P^{(0)}(x)=-\sum_{i, j=1}^{n} \frac{\partial U_{i}^{(0)}}{\partial x_{j}} \frac{\partial U_{j}^{(0)}}{\partial x_{i}},
$$

and

$$
E^{(0)}=\left(E_{1}^{(0)}, E_{2}^{(0)}, E_{3}^{(0)}, \ldots, E_{n}^{(0)}\right)^{T} .
$$

A family of explicit examples In fact, many vector functions can be chosen to satisfy (2.4)-(2.6). We now give a family of exact examples of the initial approximation function satisfying (2.4)-(2.6).

Let the integers $p>1$ and $q>2$. We choose the initial approximation functions of the form

$$
U^{(0)}(x)=\left(U_{1}^{(0)}(x), U_{2}^{(0)}(x), \ldots, U_{n}^{(0)}(x)\right), \quad \forall x \in \Omega:=([0, T])^{n},
$$

where

$$
\left\{\begin{aligned}
U_{1}^{(0)}(x) & :=\varepsilon_{0} x_{1}^{q}\left(x_{1}-T\right)^{q} g\left(x_{2}\right) g\left(x_{3}\right) e^{-r\left(x_{1}, x_{2}, x_{3}\right)}, \\
U_{2}^{(0)}(x) & :=\varepsilon_{0} x_{2}^{q}\left(x_{2}-T\right)^{q} g\left(x_{1}\right) g\left(x_{3}\right) e^{-r\left(x_{1}, x_{2}, x_{3}\right)} \\
U_{3}^{(0)}(x) & :=-2 \varepsilon_{0} x_{3}^{q}\left(x_{3}-T\right)^{q} g\left(x_{1}\right) g\left(x_{2}\right) e^{-r\left(x_{1}, x_{2}, x_{3}\right)}, \\
U_{4}^{(0)}(x) & :=0 \\
& \cdots, \\
U_{n}^{(0)}(x) & :=0
\end{aligned}\right.
$$

and 


$$
\begin{aligned}
g(y) & :=y^{q-1}(y-T)^{q-1}(2 y-T)\left(q-2(p+1) y^{2(p+1)}(y-T)^{2(p+1)}\right), \\
r\left(x_{1}, x_{2}, x_{3}\right) & :=\sum_{k=1}^{3} x_{k}^{2(p+1)}\left(x_{k}-T\right)^{2(p+1)},
\end{aligned}
$$

By direct computations, it follows that

$$
\begin{aligned}
& \partial_{x_{1}} U_{1}^{(0)}(x)=\partial_{x_{2}} U_{2}^{(0)}(x)=\varepsilon_{0} g\left(x_{1}\right) g\left(x_{2}\right) g\left(x_{3}\right) e^{-r\left(x_{1}, x_{2}, x_{3}\right)}, \\
& \partial_{x_{3}} U_{3}^{(0)}(x)=-2 \varepsilon_{0} g\left(x_{1}\right) g\left(x_{2}\right) g\left(x_{3}\right) e^{-r\left(x_{1}, x_{2}, x_{3}\right)},
\end{aligned}
$$

hence

$$
\begin{aligned}
\nabla \cdot U^{(0)}(x) & =\sum_{k=1}^{n} \partial_{x_{k}} U_{k}^{(0)}(x) \\
& =\sum_{k=1}^{3} \partial_{x_{k}} U_{k}^{(0)}(x) \\
& =0,
\end{aligned}
$$

and

$$
\left.U^{(0)}(x)\right|_{x \in \partial \Omega}=0 .
$$

Moreover, we observe that these functions decay to 0 as $|x| \rightarrow+\infty$, and for every fixed integer $p$, the assumptions (2.4)-(2.6) are fulfilled.

Here, the initial approximation pressure satisfies

$$
\Delta P^{(0)}(x)=-\sum_{i, j=1}^{n} \frac{\partial U_{i}^{(0)}}{\partial x_{j}} \frac{\partial U_{j}^{(0)}}{\partial x_{i}} .
$$

\subsection{The Carleman estimate of linearized equations}

We now construct the first approximation solution denoted by $U^{(1)}(x)$ of (2.3). The first approximation step between the initial approximation function and first approximation solution is denoted by

$$
\mathbf{h}^{(1)}(x):=U^{(1)}(x)-U^{(0)}(x) .
$$

Then we linearize the nonlinear system $(2.3)$ around $U^{(0)}$ to get the linearized operators as follows

$$
\mathcal{L}\left[U^{0}\right] \mathbf{h}^{(1)}:=-v \Delta \mathbf{h}^{(1)}+\Pi_{N_{1}}\left[\left(U^{(0)} \cdot \nabla\right) \mathbf{h}^{(1)}+\left(\mathbf{h}^{(1)} \cdot \nabla\right) U^{(0)}+\nabla\left(\mathcal{D}_{U^{(0)}} P\right) \mathbf{h}^{(1)}\right],
$$

where $\mathcal{D}_{U^{(0)}}$ denotes the Fréchet derivatives on $U^{(0)}$. By (1.3), we obtain

$$
\nabla\left(\mathcal{D}_{U^{(0)}} P\right) \mathbf{h}^{(1)}:=-\nabla \Delta^{-1} \sum_{i, j=1}^{n}\left(\partial_{x_{j}} h_{i}^{(1)} \partial_{x_{i}} U_{j}^{(0)}+\partial_{x_{j}} U_{i}^{(0)} \partial_{x_{i}} h_{j}^{(1)}\right) .
$$


We now consider the linear system

$$
\begin{aligned}
& \mathcal{L}\left[U^{0}\right] \mathbf{h}^{(1)}=\Pi_{N_{1}} E^{(0)}, \\
& \nabla \cdot \mathbf{h}^{(1)}=0,
\end{aligned}
$$

and the boundary condition

$$
\left.\mathbf{h}^{(1)}(x)\right|_{x \in \partial \Omega}=0 .
$$

The solution of this problem gives the first approximation step of the steady incompressible Navier-Stokes equations (1.1).

Before we carry out some a priori estimates, for $j=1,2,3, \ldots, n$, we rewrite equations of (2.9) into a coupled system as follows

$$
\begin{gathered}
-v \Delta h_{j}^{(1)}+\Pi_{N_{1}} \sum_{i=1}^{n} U_{i}^{(0)} \partial_{x_{i}} h_{j}^{(1)}+\Pi_{N_{1}} \sum_{i=1}^{n} h_{i}^{(1)} \partial_{x_{i}} U_{j}^{(0)} \\
+\Pi_{N_{1}} \partial_{x_{j}}\left(\left(\mathcal{D}_{U^{(0)}} P\right) \mathbf{h}^{(1)}\right)=\Pi_{N_{1}} E_{j}^{(0)}
\end{gathered}
$$

with the Dirichlet boundary condition

$$
\left.h_{j}^{(1)}(x)\right|_{x \in \partial \Omega}=0 .
$$

We now derive the Carleman-type estimate of the solution to the linear system (2.11).

Lemma 2.2 Let $v \geq 1$. Assume the initial approximation function $U^{(0)}$ satisfying (2.4)(2.6). Then the solution $\mathbf{h}^{(1)}(x)$ of the linear system (2.11) satisfies

$$
\sum_{j=1}^{n} \sum_{i=1}^{n} \int_{\Omega}\left(\partial_{x_{i}} h_{j}^{(1)}\right)^{2} d x+\Pi_{N_{1}} \sum_{j=1}^{n} \int_{\Omega}\left(h_{j}^{(1)}\right)^{2} d x \lesssim \Pi_{N_{1}} \sum_{j=1}^{n} \int_{\Omega}\left(E_{j}^{(0)}\right)^{2} d x .
$$

Proof Let $\psi\left(x_{1}\right)$ be a function with $x_{1} \in \Omega$ such that

$$
0<\kappa \leq \psi^{\prime \prime}\left(x_{1}\right)-\left(\psi^{\prime}\left(x_{1}\right)\right)^{2}-\left|\psi^{\prime}\left(x_{1}\right)\right|<\frac{1}{4},
$$

and $e^{-\psi\left(x_{1}\right)}$ is bounded for $x_{1} \in \Omega$. Here the constant $\kappa$ belongs to $\left(0, \frac{1}{4}\right)$. The condition (2.14) implies $\psi^{\prime \prime}\left(x_{1}\right)>\frac{1}{4}>0$. In fact, there are many functions satisfying these conditions. We give in what follows a simple example. Since $x_{1} \in \Omega$, there exists a suitable positive constant $b>1$ such that the function

$$
\psi\left(x_{1}\right)=2 a b x_{1}-4 b \ln \left(e^{\frac{a}{2}\left(x_{1}+b\right)}-1\right)
$$

is well defined. Here, we take the constant $a:=\left(\frac{1}{4}-\kappa\right)^{-\frac{1}{2}}$.

Direct computations give that

$$
\psi^{\prime}\left(x_{1}\right)=-2 a b\left(e^{\frac{a}{2}\left(x_{1}+b\right)}-1\right)^{-1},
$$

thus the ODE inequality (2.14) holds for a suitable $b>1$. Meanwhile, there exists a positive constant $C_{0}$ such that 


$$
e^{-\psi\left(x_{1}\right)} \leq C_{0} .
$$

Multiplying both sides of equations in (2.11) by $e^{-\psi\left(x_{1}\right)} h_{j}^{(1)}$, respectively, then integrating over $\Omega$, by noticing the Dirichlet boundary condition (2.10), for $j=1,2,3, \ldots, n$, it follows that

$$
\begin{aligned}
& v \sum_{i=1}^{n} \int_{\Omega}\left(\partial_{x_{i}} h_{j}^{(1)}\right)^{2} e^{-\psi\left(x_{1}\right)} d x+\frac{v}{2} \int_{\Omega}\left(\psi^{\prime \prime}\left(x_{1}\right)-\left(\psi^{\prime}\left(x_{1}\right)\right)^{2}\right)\left(h_{j}^{(1)}\right)^{2} e^{-\psi\left(x_{1}\right)} d x \\
& \quad+\Pi_{N_{1}} \sum_{i=1}^{n} \int_{\Omega}\left(U_{i}^{(0)} \partial_{x_{i}} h_{j}^{(1)}\right) h_{j}^{(1)} e^{-\psi\left(x_{1}\right)} d x \\
& \quad+\Pi_{N_{1}} \sum_{i=1}^{n} \int_{\Omega}\left(h_{i}^{(1)} \partial_{x_{i}} U_{j}^{(0)}\right) h_{j}^{(1)} e^{-\psi\left(x_{1}\right)} d x \\
& \quad+\Pi_{N_{1}} \int_{\Omega} \partial_{x_{j}}\left(\left(\mathcal{D}_{U^{(0)}} P\right) \mathbf{h}^{(1)}\right) h_{j}^{(1)} e^{-\psi\left(x_{1}\right)} d x \\
& =\Pi_{N_{1}} \int_{\Omega} E_{j}^{(0)} h_{j}^{(1)} e^{-\psi\left(x_{1}\right)} d x .
\end{aligned}
$$

We sum up (2.15) from $j=1$ to $j=n$, then it follows that

$$
\begin{aligned}
& v \sum_{j=1}^{n} \sum_{i=1}^{n} \int_{\Omega}\left(\partial_{x_{i}} h_{j}^{(1)}\right)^{2} e^{-\psi\left(x_{1}\right)} d x+\frac{v}{2} \sum_{j=1}^{n} \int_{\Omega}\left(\psi^{\prime \prime}\left(x_{1}\right)-\left(\psi^{\prime}\left(x_{1}\right)\right)^{2}\right)\left(h_{j}^{(1)}\right)^{2} e^{-\psi\left(x_{1}\right)} d x \\
& \quad+\Pi_{N_{1}} \sum_{j=1}^{n} \sum_{i=1}^{n} \int_{\Omega}\left(U_{i}^{(0)} \partial_{x_{i}} h_{j}^{(1)}\right) h_{j}^{(1)} e^{-\psi\left(x_{1}\right)} d x \\
& \quad+\Pi_{N_{1}} \sum_{j=1}^{n} \sum_{i=1}^{n} \int_{\Omega}\left(h_{i}^{(1)} \partial_{x_{i}} U_{j}^{(0)}\right) h_{j}^{(1)} e^{-\psi\left(x_{1}\right)} d x \\
& \quad+\Pi_{N_{1}} \sum_{j=1}^{n} \int_{\Omega} \partial_{x_{j}}\left(\left(\mathcal{D}_{U^{(0)}} P\right) \mathbf{h}^{(1)}\right) h_{j}^{(1)} e^{-\psi\left(x_{1}\right)} d x \\
& =\Pi_{N_{1}} \int_{\Omega} E_{j}^{(0)} h_{j}^{(1)} e^{-\psi\left(x_{1}\right)} d x .
\end{aligned}
$$

On the one hand, note that we have chosen the initial approximation function $U^{(0)}$ satisfying (2.4)-(2.6). We integrate by parts to get

$$
\begin{aligned}
\sum_{j=1}^{n} \sum_{i=1}^{n} \int_{\Omega}\left(U_{i}^{(0)} \partial_{x_{i}} h_{j}^{(1)}\right) h_{j}^{(1)} e^{-\psi\left(x_{1}\right)} d x= & -\frac{1}{2} \sum_{j=1}^{n} \sum_{i=1}^{n} \int_{\Omega} \partial_{x_{i}} U_{i}^{(0)}\left(h_{j}^{(1)}\right)^{2} e^{-\psi\left(x_{1}\right)} d x \\
& +\frac{1}{2} \sum_{j=1}^{n} \int_{\Omega} \psi^{\prime}\left(x_{1}\right) U_{1}^{(0)}\left(h_{j}^{(1)}\right)^{2} e^{-\psi\left(x_{1}\right)} d x,
\end{aligned}
$$

since the initial approximation function $U^{(0)}$ satisfies $\nabla \cdot U^{(0)}$, inequality $(2.17)$ is reduced into 


$$
\sum_{j=1}^{n} \sum_{i=1}^{n} \int_{\Omega}\left(U_{i}^{(0)} \partial_{x_{i}} h_{j}^{(1)}\right) h_{j}^{(1)} e^{-\psi\left(x_{1}\right)} d x=\frac{1}{2} \sum_{j=1}^{n} \int_{\Omega} \psi^{\prime}\left(x_{1}\right) U_{1}^{(0)}\left(h_{j}^{(1)}\right)^{2} e^{-\psi\left(x_{1}\right)} d x,
$$

and direct computation gives that

$$
\begin{aligned}
\sum_{j=1}^{n} \sum_{i=1}^{n} \int_{\Omega} h_{i}^{(1)} \partial_{x_{i}} U_{j}^{(0)} h_{j}^{(1)} e^{-\psi\left(x_{1}\right)} d x= & \sum_{j=1}^{n} \int_{\Omega} \partial_{x_{j}} U_{j}^{(0)}\left(h_{j}^{(1)}\right)^{2} e^{-\psi\left(x_{1}\right)} d x \\
& +\sum_{j=1}^{n} \sum_{i \neq j} \int_{\Omega} h_{i}^{(1)} \partial_{x_{i}} U_{j}^{(0)} h_{j}^{(1)} e^{-\psi\left(x_{1}\right)} d x
\end{aligned}
$$

and noticing the incompressible condition

$$
\nabla \cdot \mathbf{h}^{(1)}=0
$$

it follows that

$$
\begin{aligned}
\sum_{j=1}^{n} \int_{\Omega} \partial_{x_{j}}\left(\left(\mathcal{D}_{U^{(0)}} P\right) \mathbf{h}^{(1)}\right) h_{j}^{(1)} e^{-\psi\left(x_{1}\right)} d x= & -\sum_{j=1}^{n} \int_{\Omega}\left(\left(\mathcal{D}_{U^{(0)}} P\right) \mathbf{h}^{(1)}\right) \partial_{x_{j}} h_{j}^{(1)} e^{-\psi\left(x_{1}\right)} d x \\
& +\int_{\Omega} \psi^{\prime}\left(x_{1}\right)\left(\left(\mathcal{D}_{U^{(0)}} P\right) \mathbf{h}^{(1)}\right) h_{1}^{(1)} e^{-\psi\left(x_{1}\right)} d x \\
= & \int_{\Omega} \psi^{\prime}\left(x_{1}\right)\left(\left(\mathcal{D}_{U^{(0)}} P\right) \mathbf{h}^{(1)}\right) h_{1}^{(1)} e^{-\psi\left(x_{1}\right)} d x .
\end{aligned}
$$

Furthermore, by (2.8), using the standard Calderon-Zygmund theory and Young's inequality, it follows that

$$
\begin{aligned}
& \left|\int_{\Omega} \psi^{\prime}\left(x_{1}\right)\left(\left(\mathcal{D}_{U^{(0)}} P\right) \mathbf{h}^{(1)}\right) h_{1}^{(1)} e^{-\psi\left(x_{1}\right)} d x\right| \\
& =\left|\sum_{i=1}^{n} \sum_{j=1}^{n} \int_{\Omega} \psi^{\prime}\left(x_{1}\right)\left(\partial_{x_{j}} h_{i}^{(1)} \partial_{x_{i}} U_{j}^{(0)}+\partial_{x_{j}} U_{i}^{(0)} \partial_{x_{i}} h_{j}^{(1)}\right) h_{1}^{(1)} e^{-\psi\left(x_{1}\right)} d x\right| \\
& \lesssim \frac{1}{2} \sum_{i=1}^{n} \sum_{j=1}^{n} \int_{\Omega}\left|\psi^{\prime}\left(x_{1}\right)\left(\partial_{x_{i}} U_{j}^{(0)}+\partial_{x_{j}} U_{i}^{(0)}\right)\right|\left(h_{1}^{(1)}\right)^{2} e^{-\psi\left(x_{1}\right)} d x \\
& \quad+\frac{1}{2} \sum_{i=1}^{n} \sum_{j=1}^{n} \int_{\Omega}\left|\psi^{\prime}\left(x_{1}\right)\right|\left(\left|\partial_{x_{i}} U_{j}^{(0)}\right|\left(\partial_{x_{j}} h_{i}^{(1)}\right)^{2}+\left|\partial_{x_{j}} U_{i}^{(0)}\right|\left(\partial_{x_{i}} h_{j}^{(1)}\right)^{2}\right) e^{-\psi\left(x_{1}\right)} d x .
\end{aligned}
$$

On the other hand, by the Young inequality, we obtain

$$
\begin{aligned}
& \sum_{j=1}^{n} \sum_{i \neq j} \int_{\Omega} h_{i}^{(1)} \partial_{x_{i}} U_{j}^{(0)} h_{j}^{(1)} e^{-\psi\left(x_{1}\right)} d x \\
& \quad \leq \frac{1}{2} \sum_{j=1}^{n} \sum_{i \neq j} \int_{\Omega}\left|\partial_{x_{i}} U_{j}^{(0)}\right|\left(\left(h_{i}^{(1)}\right)^{2}+\left(h_{j}^{(1)}\right)^{2}\right) e^{-\psi\left(x_{1}\right)} d x,
\end{aligned}
$$

and 


$$
\begin{aligned}
& \sum_{j=1}^{n} \int_{\Omega} E_{j}^{(0)} h_{j}^{(1)} e^{-\psi\left(x_{1}\right)} d x \\
& \quad \leq \frac{1}{2} \sum_{j=1}^{n} \int_{\Omega}\left(\left|\left(\psi^{\prime \prime}\left(x_{1}\right)\right)^{-1}\right|\left(E_{j}^{(0)}\right)^{2}+\left|\psi^{\prime \prime}\left(x_{1}\right)\right|\left(h_{j}^{(1)}\right)^{2}\right) e^{-\psi\left(x_{1}\right)} d x .
\end{aligned}
$$

Thus we substitute (2.17)-(2.23) into (2.16) and we find

$$
\begin{gathered}
\sum_{j=1}^{n} \sum_{i=1}^{n} \int_{\Omega}\left(v-\frac{1}{2}\left|\psi^{\prime}\left(x_{1}\right)\right|\left(\left|\partial_{x_{i}} U_{j}^{(0)}\right|+\left|\partial_{x_{j}} U_{i}^{(0)}\right|\right)\right)\left(\partial_{x_{i}} h_{j}^{(1)}\right)^{2} e^{-\psi\left(x_{1}\right)} d x \\
+\Pi_{N_{1}} \sum_{j=1}^{n} \int_{\Omega} A_{j}(x)\left(h_{j}^{(1)}\right)^{2} e^{-\psi\left(x_{1}\right)} d x \\
\lesssim \Pi_{N_{1}} \sum_{j=1}^{n} \int_{\Omega}\left|\left(\psi^{\prime \prime}\left(x_{1}\right)\right)^{-1}\right|\left(E_{j}^{(0)}\right)^{2} e^{-\psi\left(x_{1}\right)} d x,
\end{gathered}
$$

where the coefficients are given by

$$
\begin{aligned}
A_{1}(x):= & \frac{v}{2}\left(\psi^{\prime \prime}\left(x_{1}\right)-\left(\psi^{\prime}\left(x_{1}\right)\right)^{2}\right)-\frac{1}{2}\left|\psi^{\prime \prime}\left(x_{1}\right)\right|+\frac{1}{2} U_{1}^{(0)} \psi^{\prime}\left(x_{1}\right)+\partial_{x_{1}} U_{1}^{(0)} \\
& -\frac{1}{2} \sum_{i=1}^{n} \sum_{j=1}^{n}\left|\psi^{\prime}\left(x_{1}\right)\left(\partial_{x_{i}} U_{j}^{(0)}+\partial_{x_{j}} U_{i}^{(0)}\right)\right|-\frac{1}{2} \sum_{j=1}^{n} \sum_{i \neq j}\left|\partial_{x_{i}} U_{j}^{(0)}\right|, \\
A_{2}(x):= & \frac{v}{2}\left(\psi^{\prime \prime}\left(x_{1}\right)-\left(\psi^{\prime}\left(x_{1}\right)\right)^{2}\right)+\frac{1}{2} U_{1}^{(0)} \psi^{\prime}\left(x_{1}\right)+\partial_{x_{2}} U_{2}^{(0)}-\frac{1}{2} \sum_{j=1}^{n} \sum_{i \neq j}\left|\partial_{x_{i}} U_{j}^{(0)}\right|-\frac{1}{2}\left|\psi^{\prime \prime}\left(x_{1}\right)\right|, \\
A_{3}(x):= & \frac{v}{2}\left(\psi^{\prime \prime}\left(x_{1}\right)-\left(\psi^{\prime}\left(x_{1}\right)\right)^{2}\right)+\frac{1}{2} U_{1}^{(0)} \psi^{\prime}\left(x_{1}\right)+\partial_{x_{3}} U_{3}^{(0)}-\frac{1}{2} \sum_{j=1}^{n} \sum_{i \neq j}\left|\partial_{x_{i}} U_{j}^{(0)}\right|-\frac{1}{2}\left|\psi^{\prime \prime}\left(x_{1}\right)\right|, \\
\quad \ldots, & \\
A_{n}(x):= & \frac{v}{2}\left(\psi^{\prime \prime}\left(x_{1}\right)-\left(\psi^{\prime}\left(x_{1}\right)\right)^{2}\right)+\frac{1}{2} U_{1}^{(0)} \psi^{\prime}\left(x_{1}\right)+\partial_{x_{n}} U_{n}^{(0)}-\frac{1}{2} \sum_{j=1}^{n} \sum_{i \neq j}\left|\partial_{x_{i}} U_{j}^{(0)}\right|-\frac{1}{2}\left|\psi^{\prime \prime}\left(x_{1}\right)\right| .
\end{aligned}
$$

Since the weighted function $\psi\left(x_{1}\right)$ satisfies (2.14), the main term of $A_{1}(x)(i=1,2,3, \ldots, n)$ is

$$
\frac{v}{2}\left(\psi^{\prime \prime}\left(x_{1}\right)-\left(\psi^{\prime}\left(x_{1}\right)\right)^{2}\right)-\frac{1}{2}\left|\psi^{\prime \prime}\left(x_{1}\right)\right|
$$

Thus, it follows that

$$
\begin{aligned}
A_{1}(x) \geq & \frac{v}{2}\left(\psi^{\prime \prime}\left(x_{1}\right)-\left(\psi^{\prime}\left(x_{1}\right)\right)^{2}\right)-\frac{1}{2}\left|\psi^{\prime \prime}\left(x_{1}\right)\right|-\frac{1}{2}\left|\psi^{\prime}\left(x_{1}\right)\right|\left\|U_{1}^{(0)}\right\|_{L^{\infty}(\Omega)}-\left\|\partial_{x_{1}} U_{1}^{(0)}\right\|_{L^{\infty}(\Omega)} \\
& -\frac{1}{2} \sum_{i=1}^{3} \sum_{j=1}^{3}\left|\psi^{\prime}\left(x_{1}\right)\right|\left(\left\|\partial_{x_{i}} U_{j}^{(0)}\right\|_{L^{\infty}}+\left\|\partial_{x_{j}} U_{i}^{(0)}\right\|_{L^{\infty}}\right)-\frac{1}{2} \sum_{j=1}^{n} \sum_{i \neq j}\left\|\partial_{x_{i}} U_{j}^{(0)}\right\|_{L^{\infty}} \\
\gtrsim & \frac{v}{2}\left(\psi^{\prime \prime}\left(x_{1}\right)-\left(\psi^{\prime}\left(x_{1}\right)\right)^{2}\right)-\frac{1}{2}\left|\psi^{\prime \prime}\left(x_{1}\right)\right|-\varepsilon n\left|\psi^{\prime}\left(x_{1}\right)\right|-\varepsilon n .
\end{aligned}
$$


Combining this estimate with (2.14) and the fact that $v \geq 1$, we deduce that there exists a positive constant $C_{\nu, \varepsilon, n}$ depending on $\nu, \varepsilon, n$ such that

$$
A_{1}(x) \gtrsim \frac{\nu}{2}\left(\psi^{\prime \prime}\left(x_{1}\right)-\left(\psi^{\prime}\left(x_{1}\right)\right)^{2}-\left|\psi^{\prime}\left(x_{1}\right)\right|\right)-\varepsilon n \geq \frac{\nu \kappa}{2}-\varepsilon n \geq C_{\nu, \varepsilon, n}>0,
$$

where $\kappa \in\left(0, \frac{1}{4}\right)$. Similarly, it follows that

$$
A_{2}(x), A_{3}(x), \ldots, A_{n}(x) \gtrsim C_{v, \varepsilon, n},
$$

and

$$
\nu-\frac{1}{2}\left|\psi^{\prime}\left(x_{1}\right)\right|\left(\left|\partial_{x_{i}} U_{j}^{(0)}\right|+\left|\partial_{x_{j}} U_{i}^{(0)}\right|\right) \gtrsim C_{\nu} .
$$

Thus, it follows from (2.24) that

$$
\begin{aligned}
& \sum_{j=1}^{n} \sum_{i=1}^{n} \int_{\Omega}\left(\partial_{x_{i}} h_{j}^{(1)}\right)^{2} e^{-\psi\left(x_{1}\right)} d x+C_{\mu, \varepsilon, n} \Pi_{N_{1}} \sum_{j=1}^{n} \int_{\Omega}\left(h_{j}^{(1)}\right)^{2} e^{-\psi\left(x_{1}\right)} d x \\
& \quad \lesssim \Pi_{N_{1}} \sum_{j=1}^{n} \int_{\Omega}\left(E_{j}^{(0)}\right)^{2} e^{-\psi\left(x_{1}\right)} d x .
\end{aligned}
$$

Combining this estimate with the fact that $e^{-\psi\left(x_{1}\right)}$ is a bounded function, we obtain

$$
\sum_{j=1}^{n} \sum_{i=1}^{n} \int_{\Omega}\left(\partial_{x_{i}} h_{j}^{(1)}\right)^{2} d x+\Pi_{N_{1}} \sum_{j=1}^{n} \int_{\Omega}\left(h_{j}^{(1)}\right)^{2} d x \lesssim \Pi_{N_{1}} \sum_{j=1}^{n} \int_{\Omega}\left(E_{j}^{(0)}\right)^{2} d x .
$$

The proof is now complete.

Furthermore, we derive the higher order derivatives estimates of elliptic equations. For a fixed constant $s \geq 1$, applying $D_{i}^{s}:=\partial_{x_{i}}^{s}(\forall i=1,2,3, \ldots, n)$ to both sides of $(2.11)$, it follows that

$$
\begin{gathered}
-v \Delta D_{i}^{s} h_{j}^{(1)}+\Pi_{N_{1}} \sum_{i=1}^{n} U_{i}^{(0)} \partial_{x_{i}} D_{i}^{s} h_{j}^{(1)}+\Pi_{N_{1}} \sum_{i=1}^{n} D_{i}^{s} h_{i}^{(1)} \partial_{x_{i}} U_{j}^{(0)} \\
+\Pi_{N_{1}} \partial_{x_{j}} D_{i}^{s}\left(\left(\mathcal{D}_{U^{(0)}} P\right) \mathbf{h}^{(1)}\right)=F_{j}, \quad \text { for } j=1,2,3, \ldots, n,
\end{gathered}
$$

with the boundary condition

$$
\left.D_{i}^{l} h_{j}^{(1)}(x)\right|_{x \in \partial \Omega}=0,
$$

where $1 \leq l \leq s$ and

$$
\begin{aligned}
F_{j}:= & \Pi_{N_{1}} D_{i}^{s} E_{j}^{(0)}-\Pi_{N_{1}} \sum_{s_{1}+s_{2}=s, 0 \leq s_{2} \leq s-1} \sum_{i=1}^{n} D_{i}^{s_{1}} U_{i}^{(0)} \partial_{x_{i}} D_{i}^{s_{2}} h_{j}^{(1)} \\
& -\Pi_{N_{1}} \sum_{s_{1}+s_{2}=s, 0 \leq s_{2} \leq s-1} \sum_{i=1}^{n}\left(D_{i}^{s_{2}} h_{i}^{(1)}\right)\left(D_{i}^{s_{1}} \partial_{x_{i}} U_{j}^{(0)}\right) .
\end{aligned}
$$

Next, we derive higher derivative estimates of solutions to the coupled system (2.11). 
Lemma 2.3 Let $v \geq 1$. Assume the initial approximation function $U^{(0)}$ satisfying (2.4)(2.6). Then the solution $\mathbf{h}^{(1)}(x)$ of the linear system (2.11) satisfies

$$
\begin{aligned}
\sum_{j=1}^{n} & \sum_{i=1}^{n} \int_{\Omega}\left(\partial_{x_{j}} D_{i}^{s} h_{j}^{(1)}\right)^{2} d x+\Pi_{N_{1}} \sum_{i=1}^{n} \sum_{j=1}^{n} \int_{\Omega}\left(D_{i}^{s} h_{j}^{(1)}\right)^{2} d x \\
& \lesssim \Pi_{N_{1}} \sum_{j=1}^{n} \sum_{i=1}^{n} \sum_{l_{0}=0}^{s} \int_{\Omega}\left(D_{i}^{l_{0}} E_{j}^{(0)}\right)^{2} d x .
\end{aligned}
$$

Proof This proof is based on the induction. Let $s=1$, by (2.27), it follows that

$$
\begin{gathered}
-v \Delta D_{i}^{1} h_{j}^{(1)}+\Pi_{N_{1}} \sum_{i=1}^{n} U_{i}^{(0)} \partial_{x_{i}} D_{i}^{1} h_{j}^{(1)}+\Pi_{N_{1}} \sum_{i=1}^{n} D_{i}^{1} h_{i}^{(1)} \partial_{x_{i}} U_{j}^{(0)} \\
+\Pi_{N_{1}} \partial_{x_{j}} D_{i}^{1}\left(\left(\mathcal{D}_{U^{(0)}} P\right) \mathbf{h}^{(1)}\right)+\Pi_{N_{1}} \sum_{i=1}^{n} D_{i}^{1} U_{i}^{(0)} \partial_{x_{i}} h_{j}^{(1)} \\
+\Pi_{N_{1}} \sum_{i=1}^{n} h_{i}^{(1)} D_{i}^{1} \partial_{x_{i}} U_{j}^{(0)}=\Pi_{N_{1}} D_{i}^{1} E_{j}^{(0)}, \quad \text { for } j=1,2,3,
\end{gathered}
$$

with the boundary condition

$$
\left.D_{i}^{1} h_{j}^{(1)}(x)\right|_{x \in \partial \Omega}=0 .
$$

Let us choose the weighted function satisfies (2.14). We multiply both sides of (2.30) by $D_{i}^{1} h_{j}^{(1)} e^{-\psi\left(x_{1}\right)}$, respectively, then integrating over $\Omega$ by noticing (2.31), and summing up those equalities from $j=1$ to $j=n$, it follows that

$$
\begin{aligned}
& v \sum_{j=1}^{n} \sum_{i=1}^{n} \int_{\Omega}\left(\partial_{x_{j}} D_{i}^{1} h_{j}^{(1)}\right)^{2} e^{-\psi\left(x_{1}\right)} d x+\frac{\nu}{2} \sum_{j=1}^{n} \int_{\Omega}\left(\psi^{\prime \prime}\left(x_{1}\right)-\left(\psi^{\prime}\left(x_{1}\right)\right)^{2}\right)\left(D_{i}^{1} h_{j}^{(1)}\right)^{2} e^{-\psi\left(x_{1}\right)} d x \\
& \quad+\Pi_{N_{1}} \sum_{j=1}^{n} \sum_{i=1}^{n} \int_{\Omega} U_{i}^{(0)}\left(\partial_{x_{i}} D_{i}^{1} h_{j}^{(1)}\right)\left(D_{i}^{1} h_{j}^{(1)}\right) e^{-\psi\left(x_{1}\right)} d x \\
& \quad+\Pi_{N_{1}} \sum_{j=1}^{n} \sum_{i=1}^{n} \int_{\Omega} D_{i}^{1} h_{i}^{(1)} \partial_{x_{i}} U_{j}^{(0)} D_{i}^{1} h_{j}^{(1)} e^{-\psi\left(x_{1}\right)} d x \\
& \quad+\Pi_{N_{1}} \sum_{j=1}^{n} \int_{\Omega} \partial_{x_{j}} D_{i}^{1}\left(\left(\mathcal{D}_{U^{(0)}} P\right) \mathbf{h}^{(1)}\right) D_{i}^{1} h_{j}^{(1)} e^{-\psi\left(x_{1}\right)} d x \\
& \quad+\Pi_{N_{1}} \sum_{a=1}^{3} I_{a}=0
\end{aligned}
$$

where 


$$
\begin{aligned}
I_{1} & :=\sum_{j=1}^{n} \sum_{i=1}^{n} \int_{\Omega} D_{i}^{1} U_{i}^{(0)} \partial_{x_{i}} h_{j}^{(1)} D_{i}^{1} h_{j}^{(1)} e^{-\psi\left(x_{1}\right)} d x, \\
I_{2} & :=\sum_{j=1}^{n} \sum_{i=1}^{n} \int_{\Omega} h_{i}^{(1)} D_{i}^{1} \partial_{x_{i}} U_{j}^{(0)} D_{i}^{1} h_{j}^{(1)} e^{-\psi\left(x_{1}\right)} d x, \\
I_{3} & :=\Pi_{N_{1}} \sum_{j=1}^{n} \int_{\Omega} D_{i}^{1} E_{j}^{(0)} D_{i}^{1} h_{j}^{(1)} e^{-\psi\left(x_{1}\right)} d x .
\end{aligned}
$$

We now estimate each term in (2.32). On the one hand, since we have chosen the initial approximation function $U^{(0)}$ satisfying (2.4)-(2.6), using the similar method of getting (2.17)-(2.20), we obtain

$$
\begin{aligned}
\sum_{j=1}^{n} \sum_{i=1}^{n} \int_{\Omega} U_{i}^{(0)}\left(\partial_{x_{i}} D_{i}^{1} h_{j}^{(1)}\right)\left(D_{i}^{1} h_{j}^{(1)}\right) e^{-\psi\left(x_{1}\right)} d x & =\frac{1}{2} \sum_{j=1}^{n} \int_{\Omega} \psi^{\prime}\left(x_{1}\right) U_{1}^{(0)}\left(D_{i}^{1} h_{j}^{(1)}\right)^{2} e^{-\psi\left(x_{1}\right)} d x \\
\sum_{j=1}^{n} \sum_{i=1}^{n} \int_{\Omega} D_{i}^{1} h_{i}^{(1)} \partial_{x_{i}} U_{j}^{(0)} D_{i}^{1} h_{j}^{(1)} e^{-\psi\left(x_{1}\right)} d x= & \sum_{j=1}^{n} \int_{\Omega} \partial_{x_{j}} U_{j}^{(0)}\left(D_{j}^{1} h_{j}^{(1)}\right)^{2} e^{-\psi\left(x_{1}\right)} d x \\
& +\sum_{j=1}^{n} \sum_{i \neq j} \int_{\Omega} \partial_{x_{i}} U_{j}^{(0)}\left(D_{i}^{1} h_{i}^{(1)}\right)\left(D_{i}^{1} h_{j}^{(1)}\right) e^{-\psi\left(x_{1}\right)} d x
\end{aligned}
$$

By the incompressible condition $\nabla \cdot \mathbf{h}^{(1)}=0$ and integrating by parts, we find

$$
\begin{aligned}
& \sum_{j=1}^{n} \int_{\Omega} \partial_{x_{j}} D_{i}^{1}\left(\left(\mathcal{D}_{U^{(0)}} P\right) \mathbf{h}^{(1)}\right) D_{i}^{1} h_{j}^{(1)} e^{-\psi\left(x_{1}\right)} d x \\
& \quad=\int_{\Omega} \psi^{\prime}\left(x_{1}\right) D_{i}^{1}\left(\left(\mathcal{D}_{U^{(0)}} P\right) \mathbf{h}^{(1)}\right)\left(D_{i}^{1} h_{1}^{(1)}\right) e^{-\psi\left(x_{1}\right)} d x,
\end{aligned}
$$

from which, by the standard Calderon-Zygmund theory and Young's inequality we find

$$
\begin{gathered}
\left|\int_{\Omega} \psi^{\prime}\left(x_{1}\right) D_{i}^{1}\left(\left(\mathcal{D}_{U^{(0)}} P\right) \mathbf{h}^{(1)}\right)\left(D_{i}^{1} h_{1}^{(1)}\right) e^{-\psi\left(x_{1}\right)} d x\right| \\
=\left|\sum_{i=1}^{n} \sum_{j=1}^{n} \int_{\Omega} \psi^{\prime}\left(x_{1}\right) D_{i}^{1}\left(\partial_{x_{j}} h_{i}^{(1)} \partial_{x_{i}} U_{j}^{(0)}+\partial_{x_{j}} U_{i}^{(0)} \partial_{x_{i}} h_{j}^{(1)}\right)\left(D_{i}^{1} h_{1}^{(1)}\right) e^{-\psi\left(x_{1}\right)} d x\right| \\
\lesssim \frac{1}{2} \sum_{i=1}^{n} \sum_{j=1}^{n} \int_{\Omega}\left|\psi^{\prime}\left(x_{1}\right)\left(\partial_{x_{i}} U_{j}^{(0)}+\partial_{x_{j}} U_{i}^{(0)}+\partial_{x_{i}} D_{i}^{1} U_{j}^{(0)}+\partial_{x_{j}} D_{i}^{1} U_{i}^{(0)}\right)\right|\left(D_{i}^{1} h_{1}^{(1)}\right)^{2} e^{-\psi\left(x_{1}\right)} d x \\
+\frac{1}{2} \sum_{i=1}^{n} \sum_{j=1}^{n} \int_{\Omega}\left|\psi^{\prime}\left(x_{1}\right)\right|\left(\left|\partial_{x_{i}} U_{j}^{(0)}\right|\left(\partial_{x_{j}} D_{i}^{1} h_{i}^{(1)}\right)^{2}+\left|\partial_{x_{j}} U_{i}^{(0)}\right|\left(\partial_{x_{i}} D_{i}^{1} h_{j}^{(1)}\right)^{2}\right) e^{-\psi\left(x_{1}\right)} d x \\
\quad+\frac{1}{2} \sum_{i=1}^{n} \sum_{j=1}^{n} \int_{\Omega}\left|\psi^{\prime}\left(x_{1}\right)\right|\left(\left|\partial_{x_{i}} D_{i}^{1} U_{j}^{(0)}\right|\left(\partial_{x_{j}} h_{i}^{(1)}\right)^{2}+\left|\partial_{x_{j}} D_{i}^{1} U_{i}^{(0)}\right|\left(\partial_{x_{i}} h_{j}^{(1)}\right)^{2}\right) e^{-\psi\left(x_{1}\right)} d x,
\end{gathered}
$$

and 


$$
\begin{aligned}
& \sum_{j=1}^{n} \sum_{i \neq j} \int_{\Omega} \partial_{x_{i}} U_{j}^{(0)}\left(D_{i}^{1} h_{i}^{(1)}\right)\left(D_{i}^{1} h_{j}^{(1)}\right) e^{-\psi\left(x_{1}\right)} d x \\
& \quad \leq \frac{1}{2} \sum_{j=1}^{n} \sum_{i \neq j} \int_{\Omega}\left|\partial_{x_{i}} U_{j}^{(0)}\right|\left(\left(D_{i}^{1} h_{i}^{(1)}\right)^{2}+\left(D_{i}^{1} h_{j}^{(1)}\right)^{2}\right) e^{-\psi\left(x_{1}\right)} d x .
\end{aligned}
$$

On the other hand, it follows that

$$
\begin{gathered}
I_{1}=\sum_{j=1}^{n} \sum_{i=1}^{n} \int_{\Omega} D_{i}^{1} U_{i}^{(0)}\left(D_{i}^{1} h_{j}^{(1)}\right)^{2} e^{-\psi\left(x_{1}\right)} d x \\
I_{2} \leq \frac{1}{2} \sum_{j=1}^{n} \int_{\Omega}\left(\sum_{i=1}^{n}\left|D_{i}^{1} \partial_{x_{i}} U_{j}^{(0)}\right|\right)\left(h_{j}^{(1)}\right)^{2} e^{-\psi\left(x_{1}\right)} d x+\sum_{j=1}^{n} \int_{\Omega}\left(\sum_{i=1}^{n}\left|D_{i}^{1} \partial_{x_{i}} U_{j}^{(0)}\right|\right)\left(D_{i}^{1} h_{j}^{(1)}\right)^{2} e^{-\psi\left(x_{1}\right)} d x \\
\lesssim \varepsilon \sum_{j=1}^{n} \int_{\Omega}\left(h_{j}^{(1)}\right)^{2} e^{-\psi\left(x_{1}\right)} d x+\sum_{j=1}^{n} \int_{\Omega}\left(\sum_{i=1}^{n}\left|D_{i}^{1} \partial_{x_{i}} U_{j}^{(0)}\right|\right)\left(D_{i}^{1} h_{j}^{(1)}\right)^{2} e^{-\psi\left(x_{1}\right)} d x \\
I_{3} \leq \frac{1}{2} \sum_{j=1}^{n} \int_{\Omega}\left(\left|\left(\psi^{\prime \prime}\left(x_{1}\right)\right)^{-1}\right|\left(D_{i}^{1} E_{j}^{(0)}\right)^{2}+\left|\psi^{\prime \prime}\left(x_{1}\right)\right|\left(D_{i}^{1} h_{j}^{(1)}\right)^{2}\right) e^{-\psi\left(x_{1}\right)} d x
\end{gathered}
$$

Thus, summing up (2.32) from $i=1$ to $i=n$, we use (2.33)-(2.40) to derive

$$
\begin{aligned}
& n \sum_{j=1}^{n} \sum_{i=1}^{n} \int_{\Omega}\left(v-\frac{1}{2}\left|\psi^{\prime}\left(x_{1}\right)\right|\left(\left|\partial_{x_{i}} U_{j}^{(0)}\right|+\left|\partial_{x_{j}} U_{i}^{(0)}\right|\right)\right)\left(\partial_{x_{j}} D_{i}^{1} h_{j}^{(1)}\right)^{2} e^{-\psi\left(x_{1}\right)} d x \\
& \quad+n \Pi_{N_{1}} \sum_{j=1}^{n} \sum_{i=1}^{n} \int_{\Omega} \bar{A}_{j}(x)\left(D_{i}^{1} h_{j}^{(1)}\right)^{2} e^{-\psi\left(x_{1}\right)} d x \\
& \lesssim \Pi_{N_{1}} \sum_{j=1}^{n} \sum_{i=1}^{n} \int_{\Omega}\left|\left(\psi^{\prime \prime}\left(x_{1}\right)\right)^{-1}\right|\left(D_{i}^{1} E_{j}^{(0)}\right)^{2} e^{-\psi\left(x_{1}\right)} d x+\varepsilon \sum_{j=1}^{n} \int_{\Omega}\left(h_{j}^{(1)}\right)^{2} e^{-\psi\left(x_{1}\right)} d x \\
& \quad+\frac{1}{2} \Pi_{N_{1}} \sum_{i=1}^{n} \sum_{j=1}^{n} \int_{\Omega}\left|\psi^{\prime}\left(x_{1}\right)\right|\left(\left|\partial_{x_{i}} D_{i}^{1} U_{j}^{(0)}\right|\left(\partial_{x_{j}} h_{i}^{(1)}\right)^{2}+\left|\partial_{x_{j}} D_{i}^{1} U_{i}^{(0)}\right|\left(\partial_{x_{i}} h_{j}^{(1)}\right)^{2}\right) e^{-\psi\left(x_{1}\right)} d x,
\end{aligned}
$$

where 


$$
\begin{aligned}
\bar{A}_{1}(x):= & \frac{v}{2}\left(\psi^{\prime \prime}\left(x_{1}\right)-\left(\psi^{\prime}\left(x_{1}\right)\right)^{2}\right)+\frac{1}{2} U_{1}^{(0)} \psi^{\prime}\left(x_{1}\right)+\partial_{x_{1}} U_{1}^{(0)} \\
& -\frac{1}{2} \sum_{i=1}^{n} \sum_{j=1}^{n}\left|\psi^{\prime}\left(x_{1}\right)\left(\partial_{x_{i}} U_{j}^{(0)}+\partial_{x_{j}} U_{i}^{(0)}+\partial_{x_{i}} D_{i}^{1} U_{j}^{(0)}+\partial_{x_{j}} D_{i}^{1} U_{i}^{(0)}\right)\right| \\
& -\frac{1}{2} \sum_{j=1}^{n} \sum_{i \neq j}\left|\partial_{x_{i}} U_{j}^{(0)}\right|-\frac{1}{2}\left|\psi^{\prime \prime}\left(x_{1}\right)\right|+\sum_{i=1}^{n} D_{i}^{1} U_{i}^{(0)}-\sum_{i=1}^{n}\left|D_{i}^{1} \partial_{x_{i}} U_{j}^{(0)}\right|, \\
\bar{A}_{2}(x):= & \frac{v}{2}\left(\psi^{\prime \prime}\left(x_{1}\right)-\left(\psi^{\prime}\left(x_{1}\right)\right)^{2}\right)+\frac{1}{2} U_{1}^{(0)} \psi^{\prime}\left(x_{1}\right)+\partial_{x_{2}} U_{2}^{(0)} \\
& -\frac{1}{2} \sum_{j=1}^{n} \sum_{i \neq j}\left|\partial_{x_{i}} U_{j}^{(0)}\right|-\frac{1}{2}\left|\psi^{\prime \prime}\left(x_{1}\right)\right|+\sum_{i=1}^{n} D_{i}^{1} U_{i}^{(0)}-\sum_{i=1}^{n}\left|D_{i}^{1} \partial_{x_{i}} U_{j}^{(0)}\right|, \\
\bar{A}_{3}(x):= & \frac{v}{2}\left(\psi^{\prime \prime}\left(x_{1}\right)-\left(\psi^{\prime}\left(x_{1}\right)\right)^{2}\right)+\frac{1}{2} U_{1}^{(0)} \psi^{\prime}\left(x_{1}\right)+\partial_{x_{3}} U_{3}^{(0)} \\
& -\frac{1}{2} \sum_{j=1}^{n} \sum_{i \neq j}\left|\partial_{x_{i}} U_{j}^{(0)}\right|-\frac{1}{2}\left|\psi^{\prime \prime}\left(x_{1}\right)\right|+\sum_{i=1}^{n} D_{i}^{1} U_{i}^{(0)}-\sum_{i=1}^{n}\left|D_{i}^{1} \partial_{x_{i}} U_{j}^{(0)}\right|, \\
\ldots & -\frac{1}{2} \sum_{j=1}^{n} \sum_{i \neq j}\left|\partial_{x_{i}} U_{j}^{(0)}\right|-\frac{1}{2}\left|\psi^{\prime \prime}\left(x_{1}\right)\right|+\sum_{i=1}^{n} D_{i}^{1} U_{i}^{(0)}-\sum_{i=1}^{n}\left|D_{i}^{1} \partial_{x_{i}} U_{j}^{(0)}\right| \\
\bar{A}_{n}(x):= & \frac{v}{2}\left(\psi^{\prime \prime}\left(x_{1}\right)-\left(\psi^{\prime}\left(x_{1}\right)\right)^{2}\right)+\frac{1}{2} U_{1}^{(0)} \psi^{\prime}\left(x_{1}\right)+\partial_{x_{n}} U_{n}^{(0)} \\
&
\end{aligned}
$$

We notice that the main term of $\bar{A}_{i}(x)(i=1,2,3, \ldots, n)$ is given by

$$
\frac{v}{2}\left(\psi^{\prime \prime}\left(x_{1}\right)-\left(\psi^{\prime}\left(x_{1}\right)\right)^{2}\right)-\frac{1}{2}\left|\psi^{\prime \prime}\left(x_{1}\right)\right| .
$$

Combining this fact with the assumption (2.5), we deduce that

$$
\bar{A}_{1}(x) \gtrsim \frac{v}{2}\left(\psi^{\prime \prime}\left(x_{1}\right)-\left(\psi^{\prime}\left(x_{1}\right)\right)^{2}\right)-\frac{1}{2}\left|\psi^{\prime \prime}\left(x_{1}\right)\right|-\varepsilon n\left|\psi^{\prime}\left(x_{1}\right)\right|-\varepsilon n .
$$

Thus, by (2.14), there exists a positive constant $C_{v, \varepsilon, n}$ depending on $v, \varepsilon, n$ such that

$$
\bar{A}_{1}(x) \gtrsim \frac{v}{2}\left(\psi^{\prime \prime}\left(x_{1}\right)-\left(\psi^{\prime}\left(x_{1}\right)\right)^{2}-\left|\psi^{\prime}\left(x_{1}\right)\right|\right)-\varepsilon n \geq C_{\nu, \varepsilon, n}>0,
$$

where $\kappa \in\left(0, \frac{1}{4}\right)$. Similarly, it follows that

$$
\bar{A}_{2}(x), \bar{A}_{3}(x), \ldots, \bar{A}_{n}(x) \gtrsim C_{v, \varepsilon, n},
$$

and

$$
v-\frac{1}{2}\left|\psi^{\prime}\left(x_{1}\right)\right|\left(\left|\partial_{x_{i}} U_{j}^{(0)}\right|+\left|\partial_{x_{j}} U_{i}^{(0)}\right|\right) \gtrsim C_{v} .
$$

Therefore 


$$
\begin{aligned}
\sum_{j=1}^{n} & \sum_{i=1}^{n} \int_{\Omega}\left(\partial_{x_{j}} D_{i}^{1} h_{j}^{(1)}\right)^{2} e^{-\psi\left(x_{1}\right)} d x+C_{\nu, \varepsilon, n} \Pi_{N_{1}} \sum_{j=1}^{n} \sum_{i=1}^{n} \int_{\Omega}\left(D_{i}^{1} h_{j}^{(1)}\right)^{2} e^{-\psi\left(x_{1}\right)} d x \\
\lesssim & \Pi_{N_{1}} \sum_{j=1}^{n} \sum_{i=1}^{n} \int_{\Omega}\left(D_{i}^{1} E_{j}^{(0)}\right)^{2} e^{-\psi\left(x_{1}\right)} d x+\varepsilon \sum_{j=1}^{n} \int_{\Omega}\left(h_{j}^{(1)}\right)^{2} e^{-\psi\left(x_{1}\right)} d x \\
& +\Pi_{N_{1}} \sum_{i=1}^{n} \sum_{j=1}^{n} \int_{\Omega}\left(\left(\partial_{x_{j}} h_{i}^{(1)}\right)^{2}+\left(\partial_{x_{i}} h_{j}^{(1)}\right)^{2}\right) e^{-\psi\left(x_{1}\right)} d x .
\end{aligned}
$$

Furthermore, one can see that the last two terms in the right-hand side of (2.42) can be controlled by using (2.13). Thus, it follows that

$$
\begin{aligned}
\sum_{j=1}^{n} & \sum_{i=1}^{n} \int_{\Omega}\left(\partial_{x_{j}} D_{i}^{1} h_{j}^{(1)}\right)^{2} e^{-\psi\left(x_{1}\right)} d x+\Pi_{N_{1}} \sum_{j=1}^{n} \sum_{i=1}^{n} \int_{\Omega}\left(D_{i}^{1} h_{j}^{(1)}\right)^{2} e^{-\psi\left(x_{1}\right)} d x \\
& \lesssim \Pi_{N_{1}} \sum_{j=1}^{n} \sum_{i=1}^{n} \int_{\Omega}\left(D_{i}^{1} E_{j}^{(0)}\right)^{2} e^{-\psi\left(x_{1}\right)} d x+\Pi_{N_{1}} \sum_{j=1}^{n} \int_{\Omega}\left(E_{j}^{(0)}\right)^{2} e^{-\psi\left(x_{1}\right)} d x .
\end{aligned}
$$

Assume that the $2 \leq l \leq s-1$ derivative case holds, that is,

$$
\begin{aligned}
& \sum_{j=1}^{n} \sum_{i=1}^{n} \int_{\Omega}\left(\partial_{x_{j}} D_{i}^{l} h_{j}^{(1)}\right)^{2} e^{-\psi\left(x_{1}\right)} d x+\Pi_{N_{1}} \sum_{i=1}^{n} \sum_{j=1}^{n} \int_{\Omega}\left(D_{i}^{l} h_{j}^{(1)}\right)^{2} e^{-\psi\left(x_{1}\right)} d x \\
& \quad \lesssim \Pi_{N_{1}} \sum_{j=1}^{n} \sum_{i=1}^{n} \sum_{l_{0}=0}^{l} \int_{\Omega}\left(D_{i}^{l_{0}} E_{j}^{(0)}\right)^{2} e^{-\psi\left(x_{1}\right)} d x .
\end{aligned}
$$

We now prove that the $s$ th derivative case holds. Multiplying both sides of equations (2.27) by $D_{i}^{s} h_{j}^{(1)} e^{-\psi\left(x_{1}\right)}$, then integrating over $\Omega$ by using the boundary condition (2.28), and summing up those equalities from $j=1$ to $j=n$, it follows that

$$
\begin{aligned}
& v \sum_{j=1}^{n} \sum_{i=1}^{n} \int_{\Omega}\left(\partial_{x_{j}} D_{i}^{s} h_{j}^{(1)}\right)^{2} e^{-\psi\left(x_{1}\right)} d x+\frac{v}{2} \sum_{j=1}^{n} \int_{\Omega}\left(\psi^{\prime \prime}\left(x_{1}\right)-\left(\psi^{\prime}\left(x_{1}\right)\right)^{2}\right)\left(D_{i}^{s} h_{j}^{(1)}\right)^{2} e^{-\psi\left(x_{1}\right)} d x \\
& \quad+\Pi_{N_{1}} \sum_{j=1}^{n} \sum_{i=1}^{n} \int_{\Omega} U_{i}^{(0)}\left(\partial_{x_{i}} D_{i}^{s} h_{j}^{(1)}\right)\left(D_{i}^{s} h_{j}^{(1)}\right) e^{-\psi\left(x_{1}\right)} d x \\
& \quad+\Pi_{N_{1}} \sum_{j=1}^{n} \sum_{i=1}^{n} \int_{\Omega} D_{i}^{s} h_{i}^{(1)} \partial_{x_{i}} U_{j}^{(0)} D_{i}^{s} h_{j}^{(1)} e^{-\psi\left(x_{1}\right)} d x \\
& \quad+\Pi_{N_{1}} \sum_{j=1}^{n} \int_{\Omega} \partial_{x_{j}} D_{i}^{s}\left(\left(\mathcal{D}_{U^{(0)}} P\right) \mathbf{h}^{(1)}\right) D_{i}^{s} h_{j}^{(1)} e^{-\psi\left(x_{1}\right)} d x \\
& =\sum_{j=1}^{n} \int_{\Omega} F_{j} D_{i}^{s} h_{j}^{(1)} e^{-\psi\left(x_{1}\right)} d x .
\end{aligned}
$$

We notice that 


$$
\begin{aligned}
\mid \int_{\Omega} & \psi^{\prime}\left(x_{1}\right) D_{i}^{s}\left(\left(\mathcal{D}_{U^{(0)}} P\right) \mathbf{h}^{(1)}\right)\left(D_{i}^{s} h_{1}^{(1)}\right) e^{-\psi\left(x_{1}\right)} d x \mid \\
= & \left|\sum_{i=1}^{n} \sum_{j=1}^{n} \int_{\Omega} \psi^{\prime}\left(x_{1}\right) D_{i}^{s}\left(\partial_{x_{j}} h_{i}^{(1)} \partial_{x_{i}} U_{j}^{(0)}+\partial_{x_{j}} U_{i}^{(0)} \partial_{x_{i}} h_{j}^{(1)}\right)\left(D_{i}^{s} h_{1}^{(1)}\right) e^{-\psi\left(x_{1}\right)} d x\right| \\
= & \mid \sum_{i=1}^{n} \sum_{j=1}^{n} \int_{\Omega} \psi^{\prime}\left(x_{1}\right) \sum_{j_{1}+j_{2}=s, 0 \leq j_{1} j_{2} \leq s}\left(D_{i}^{j_{2}} \partial_{x_{j}} h_{i}^{(1)} D_{i}^{j_{1}} \partial_{x_{i}} U_{j}^{(0)}\right. \\
& \left.+D_{i}^{j_{1}} \partial_{x_{j}} U_{i}^{(0)} D_{i}^{j_{2}} \partial_{x_{i}} h_{j}^{(1)}\right)\left(D_{i}^{s} h_{1}^{(1)}\right) e^{-\psi\left(x_{1}\right)} d x \mid \\
\lesssim & \frac{1}{2} \sum_{i=1}^{n} \sum_{j=1}^{n} \sum_{j_{1}=1}^{s} \int_{\Omega}\left|\psi^{\prime}\left(x_{1}\right)\left(\partial_{x_{i}} D_{i}^{j_{1}} U_{j}^{(0)}+\partial_{x_{j}} D_{i}^{j_{1}} U_{i}^{(0)}\right)\right|\left(D_{i}^{s} h_{1}^{(1)}\right)^{2} e^{-\psi\left(x_{1}\right)} d x \\
+ & \frac{1}{2} \sum_{i=1}^{n} \sum_{j=1}^{n} \sum_{j_{1}+j_{2}=s, 0 \leq j_{2} \leq s-1} \int_{\Omega}\left|\psi^{\prime}\left(x_{1}\right)\right|\left(\left|\partial_{x_{i}} D_{i}^{j_{1}} U_{j}^{(0)}\right|\left(\partial_{x_{j}} D_{i}^{j_{2}} h_{i}^{(1)}\right)^{2}\right. \\
& \left.+\left|\partial_{x_{j}} D_{i}^{j_{1}} U_{i}^{(0)}\right|\left(\partial_{x_{i}} D_{i}^{j_{2}} h_{j}^{(1)}\right)^{2}\right) e^{-\psi\left(x_{1}\right)} d x \\
& +\frac{1}{2} \sum_{i=1}^{n} \sum_{j=1}^{n} \int_{\Omega}\left|\psi^{\prime}\left(x_{1}\right)\right|\left(\left|\partial_{x_{i}} U_{j}^{(0)}\right|\left(\partial_{x_{j}} D_{i}^{s} h_{i}^{(1)}\right)^{2}+\left|\partial_{x_{j}} U_{i}^{(0)}\right|\left(\partial_{x_{i}} D_{i}^{s} h_{j}^{(1)}\right)^{2}\right) e^{-\psi\left(x_{1}\right)} d x
\end{aligned}
$$

and

$$
\begin{aligned}
& \sum_{s_{1}+s_{2}=s, 0 \leq s_{2} \leq s-1} \sum_{j=1}^{n} \sum_{i=1}^{n} \int_{\Omega} D_{i}^{s_{1}} U_{i}^{(0)}\left(\partial_{x_{i}} D_{i}^{s_{2}} h_{j}^{(1)}\right) D_{i}^{s} h_{j}^{(1)} e^{-\psi\left(x_{1}\right)} d x \\
= & \sum_{s_{1}+s_{2}=s, 0 \leq s_{2} \leq s-2} \sum_{j=1}^{n} \sum_{i=1}^{n} \int_{\Omega} D_{i}^{s_{1}} U_{i}^{(0)}\left(\partial_{x_{i}} D_{i}^{s_{2}} h_{j}^{(1)}\right) D_{i}^{s} h_{j}^{(1)} e^{-\psi\left(x_{1}\right)} d x \\
& +\sum_{j=1}^{n} \sum_{i=1}^{n} \int_{\Omega} D_{i}^{1} U_{i}^{(0)}\left(D_{i}^{s} h_{j}^{(1)}\right)^{2} e^{-\psi\left(x_{1}\right)} d x .
\end{aligned}
$$

Thus, with similar arguments as for getting (2.41), we can obtain 


$$
\begin{aligned}
n \sum_{j=1}^{n} & \sum_{i=1}^{n} \int_{\Omega}\left(v-\frac{1}{2}\left|\psi^{\prime}\left(x_{1}\right)\right|\left(\left|\partial_{x_{i}} U_{j}^{(0)}\right|+\left|\partial_{x_{j}} U_{i}^{(0)}\right|\right)\right)\left(\partial_{x_{j}} D_{i}^{s} h_{j}^{(1)}\right)^{2} e^{-\psi\left(x_{1}\right)} d x \\
& +n \Pi_{N_{1}} \sum_{j=1}^{n} \sum_{i=1}^{n} \int_{\Omega} C_{j}(x)\left(D_{i}^{s} h_{j}^{(1)}\right)^{2} e^{-\psi\left(x_{1}\right)} d x \\
\lesssim & \Pi_{N_{1}} \sum_{j=1}^{n} \sum_{i=1}^{n} \int_{\Omega}\left|\left(\psi^{\prime \prime}\left(x_{1}\right)\right)^{-1}\right|\left(D_{i}^{s} E_{j}^{(0)}\right)^{2} e^{-\psi\left(x_{1}\right)} d x \\
& +\Pi_{N_{1}} \sum_{s_{1}+s_{2}=s, 0 \leq s_{2} \leq s-1} \sum_{i=1}^{n} \int_{\Omega}\left(D_{i}^{s_{1}} \partial_{x_{i}} U_{j}^{(0)}\right)\left(D_{i}^{s_{2}} h_{i}^{(1)}\right)^{2} e^{-\psi\left(x_{1}\right)} d x \\
& +\frac{1}{2} \sum_{i=1}^{n} \sum_{j=1}^{n} \sum_{j_{1}+j_{2}=s, 0 \leq j_{2} \leq s-1} \int_{\Omega}\left|\psi^{\prime}\left(x_{1}\right)\right|\left(\left|\partial_{x_{i}} D_{i}^{j_{1}} U_{j}^{(0)}\right|\left(\partial_{x_{j}} D_{i}^{j_{2}} h_{i}^{(1)}\right)^{2}\right. \\
& \left.+\left|\partial_{x_{j}} D_{i}^{j_{1}} U_{i}^{(0)}\right|\left(\partial_{x_{i}} D_{i}^{j_{2}} h_{j}^{(1)}\right)^{2}\right) e^{-\psi\left(x_{1}\right)} d x,
\end{aligned}
$$

where

$$
\begin{aligned}
C_{1}(x):= & \frac{v}{2}\left(\psi^{\prime \prime}\left(x_{1}\right)-\left(\psi^{\prime}\left(x_{1}\right)\right)^{2}\right)+\frac{1}{2} U_{1}^{(0)} \psi^{\prime}\left(x_{1}\right)+\partial_{x_{1}} U_{1}^{(0)}+\sum_{i=1}^{n} D_{i}^{1} U_{i}^{(0)} \\
& -\frac{1}{2} \sum_{j_{1}=1}^{s}\left|\psi^{\prime}\left(x_{1}\right)\left(\partial_{x_{i}} D_{i}^{j_{1}} U_{j}^{(0)}+\partial_{x_{j}} D_{i}^{j_{1}} U_{i}^{(0)}\right)\right|-\frac{1}{2}\left|\psi^{\prime}\left(x_{1}\right)\right| \sum_{i=1}^{n}\left|D_{i}^{2} U_{i}^{(0)}\right| \\
& -\frac{1}{2} \sum_{j=1}^{n} \sum_{i \neq j}\left|\partial_{x_{i}} U_{j}^{(0)}\right|-\frac{1}{2}\left|\psi^{\prime \prime}\left(x_{1}\right)\right|-\sum_{i=1}^{n} \sum_{j=1}^{n}\left|D_{i}^{j} \partial_{x_{i}} U_{j}^{(0)}\right|, \\
C_{2}(x):= & \frac{v}{2}\left(\psi^{\prime \prime}\left(x_{1}\right)-\left(\psi^{\prime}\left(x_{1}\right)\right)^{2}\right)+\frac{1}{2} U_{1}^{(0)} \psi^{\prime}\left(x_{1}\right)+\partial_{x_{2}} U_{2}^{(0)}+\sum_{i=1}^{n} D_{i}^{1} U_{i}^{(0)} \\
& -\frac{1}{2} \sum_{j=1}^{n} \sum_{i \neq j}\left|\partial_{x_{i}} U_{j}^{(0)}\right|-\frac{1}{2}\left|\psi^{\prime \prime}\left(x_{1}\right)\right|-\frac{1}{2}\left|\psi^{\prime}\left(x_{1}\right)\right| \sum_{i=1}^{n}\left|D_{i}^{2} U_{i}^{(0)}\right|-\sum_{i=1}^{n} \sum_{j=1}^{n}\left|D_{i}^{j} \partial_{x_{i}} U_{j}^{(0)}\right|, \\
C_{3}(x):= & \frac{v}{2}\left(\psi^{\prime \prime}\left(x_{1}\right)-\left(\psi^{\prime}\left(x_{1}\right)\right)^{2}\right)+\frac{1}{2} U_{1}^{(0)} \psi^{\prime}\left(x_{1}\right)+\partial_{x_{3}} U_{3}^{(0)}+\sum_{i=1}^{n} D_{i}^{1} U_{i}^{(0)} \\
& -\frac{1}{2} \sum_{j=1}^{n} \sum_{i \neq j}\left|\partial_{x_{i}} U_{j}^{(0)}\right|-\frac{1}{2}\left|\psi^{\prime \prime}\left(x_{1}\right)\right|-\frac{1}{2}\left|\psi^{\prime}\left(x_{1}\right)\right| \sum_{i=1}^{n}\left|D_{i}^{2} U_{i}^{(0)}\right|-\sum_{i=1}^{n} \sum_{j=1}^{n}\left|D_{i}^{j} \partial_{x_{i}} U_{j}^{(0)}\right|, \\
& \ldots \\
C_{n}(x):= & \frac{v}{2}\left(\psi^{\prime \prime}\left(x_{1}\right)-\left(\psi^{\prime}\left(x_{1}\right)\right)^{2}\right)+\frac{1}{2} U_{1}^{(0)} \psi^{\prime}\left(x_{1}\right)+\partial_{x_{n}} U_{n}^{(0)}+\sum_{i=1}^{n} D_{i}^{1} U_{i}^{(0)} \\
& -\frac{1}{2} \sum_{j=1}^{n} \sum_{i \neq j}\left|\partial_{x_{i}} U_{j}^{(0)}\right|-\frac{1}{2}\left|\psi^{\prime \prime}\left(x_{1}\right)\right|-\frac{1}{2}\left|\psi^{\prime}\left(x_{1}\right)\right| \sum_{i=1}^{n}\left|D_{i}^{2} U_{i}^{(0)}\right|-\sum_{i=1}^{n} \sum_{j=1}^{n}\left|D_{i}^{j} \partial_{x_{i}} U_{j}^{(0)}\right| .
\end{aligned}
$$

By assumptions of (2.4)-(2.5), one can see that the coefficients $C_{i}(x)(i=1,2,3, \ldots, n)$ have the same main terms with $\bar{A}_{i}(x)$. It follows that

$$
C_{1}(x) \gtrsim \frac{\nu}{2}\left(\psi^{\prime \prime}\left(x_{1}\right)-\left(\psi^{\prime}\left(x_{1}\right)\right)^{2}\right)-\frac{1}{2}\left|\psi^{\prime \prime}\left(x_{1}\right)\right|-\varepsilon\left|\psi^{\prime}\left(x_{1}\right)\right|-\varepsilon n .
$$


By this estimate and (2.14), one can deduce that there exists a positive constant $C_{v, \varepsilon, n}$ depending on $v, \varepsilon, n$ such that

$$
C_{1}(x) \gtrsim \frac{\nu}{2}\left(\psi^{\prime \prime}\left(x_{1}\right)-\left(\psi^{\prime}\left(x_{1}\right)\right)^{2}-\left|\psi^{\prime}\left(x_{1}\right)\right|\right)-\varepsilon n \geq C_{\nu, \varepsilon, n}>0,
$$

where $\kappa \in\left(0, \frac{1}{4}\right)$. Similarly, it follows that

$$
C_{2}(x), C_{3}(x), \ldots, C_{n}(x) \gtrsim C_{v, \varepsilon} .
$$

Thus, we can reduce (2.48) into

$$
\begin{aligned}
& \sum_{i=1}^{n} \int_{\Omega}\left(\partial_{x_{j}} D_{i}^{s} h_{j}^{(1)}\right)^{2} e^{-\psi\left(x_{1}\right)} d x+C_{v, \varepsilon, n} \Pi_{N_{1}} \sum_{j=1}^{n} \int_{\Omega}\left(D_{i}^{s} h_{j}^{(1)}\right)^{2} e^{-\psi\left(x_{1}\right)} d x \\
& \lesssim \Pi_{N_{1}} \int_{\Omega}\left(D_{i}^{s} E_{j}^{(0)}\right)^{2} e^{-\psi\left(x_{1}\right)} d x+\Pi_{N_{1}} \sum_{s_{1}+s_{2}=s, 0 \leq s_{2} \leq s-1} \sum_{i=1}^{n} \int_{\Omega}\left(D_{i}^{s_{1}} \partial_{x_{i}} U_{j}^{(0)}\right)\left(D_{i}^{s_{2}} h_{i}^{(1)}\right)^{2} e^{-\psi\left(x_{1}\right)} d x \\
& \quad+\frac{1}{2} \sum_{i=1}^{n} \sum_{j=1}^{n} \sum_{j_{1}+j_{2}=s, 0 \leq j_{2} \leq s-1} \int_{\Omega}\left|\psi^{\prime}\left(x_{1}\right)\right|\left(\left|\partial_{x_{i}} D_{i}^{j_{1}} U_{j}^{(0)}\right|\left(\partial_{x_{j}} D_{i}^{j_{2}} h_{i}^{(1)}\right)^{2}\right. \\
& \left.\quad+\left|\partial_{x_{j}} D_{i}^{j_{1}} U_{i}^{(0)}\right|\left(\partial_{x_{i}} D_{i}^{j_{2}} h_{j}^{(1)}\right)^{2}\right) e^{-\psi\left(x_{1}\right)} d x .
\end{aligned}
$$

Hence, with similar arguments as for getting (2.43), we use (2.44) to derive

$$
\begin{aligned}
& \sum_{j=1}^{n} \sum_{i=1}^{n} \int_{\Omega}\left(\partial_{x_{j}} D_{i}^{s} h_{j}^{(1)}\right)^{2} e^{-\psi\left(x_{1}\right)} d x+\Pi_{N_{1}} \sum_{i=1}^{n} \sum_{j=1}^{n} \int_{\Omega}\left(D_{i}^{s} h_{j}^{(1)}\right)^{2} e^{-\psi\left(x_{1}\right)} d x \\
& \quad \lesssim \Pi_{N_{1}} \sum_{j=1}^{n} \sum_{i=1}^{n} \sum_{l_{0}=0}^{s} \int_{\Omega}\left(D_{i}^{l_{0}} E_{j}^{(0)}\right)^{2} e^{-\psi\left(x_{1}\right)} d x .
\end{aligned}
$$

Combining this estimate with the fact that $e^{-\psi\left(x_{1}\right)}$ is bounded, we obtain (2.29).

\subsection{The existence of first approximation step}

Based on the previous a priori estimates, we are ready to prove the existence of the first approximation step, by using the classical theory of elliptic equations; see[16, 30].

Proposition 2.1 Assume the initial approximation function $U^{(0)}$ satisfying (2.4)-(2.6). Then the linearized elliptic system

$$
\left\{\begin{array}{l}
\mathcal{L}\left[U^{0}\right] \mathbf{h}^{(1)}=\Pi_{N_{1}} E^{(0)}, \\
\nabla \cdot \mathbf{h}^{(1)}=0, \\
\left.\mathbf{h}^{(1)}(x)\right|_{x \in \partial \Omega}=0,
\end{array}\right.
$$

admits a Sobolev regular solution $\mathbf{h}^{(1)}(x) \in H^{s}(\Omega)$.

Moreover, it follows that 


$$
\left\|\mathbf{h}^{(1)}\right\|_{H^{s}}^{2} \lesssim\left\|\Pi_{N_{1}} E^{(0)}\right\|_{H^{s}}^{2}, \quad \forall t>0
$$

Proof Let $\mathbb{P}$ be the Leray projector onto the space of divergence free functions. We apply the Leray projector to equations (2.9), it follows that

$$
-v \Delta \mathbf{h}^{(1)}+\mathbb{P} \Pi_{N_{1}}\left[\left(U^{(0)} \cdot \nabla\right) \mathbf{h}^{(1)}+\left(\mathbf{h}^{(1)} \cdot \nabla\right) U^{(0)}\right]=\mathbb{P} \Pi_{N_{1}} E^{(0)}
$$

By (2.13) in Lemma 2.2 and (2.29) in Lemma 2.3, we can get the uniform bound estimate

$$
\left\|\mathbf{h}^{(1)}\right\|_{H^{s}}^{2} \lesssim\left\|\Pi_{N_{1}} E^{(0)}\right\|_{H^{s}}^{2} .
$$

From the standard theory of elliptic equations of the general order[16, 30], the linear elliptic equations (2.51) admit a unique weak solution $\mathbf{h}^{(1)} \in H^{1}$ if $E^{(0)} \in H^{1}$. Since the error term $E^{(0)} \in H^{s}(\Omega)$ for $s>1$, we conclude that $\mathbf{h}^{(1)} \in H^{s}(\Omega)$.

\section{The $m$ th approximation step}

We define

$$
\mathcal{B}_{\varepsilon}:=\left\{u^{(k)}(x):\left\|U^{(k)}\right\|_{H^{s}} \lesssim \varepsilon<1\right\}
$$

with the integer $2 \leq k \leq m-1$ and the constant $s \geq 1$.

Assume that the $m$-th approximation solutions of (2.3) is denoted by $\mathbf{h}^{(m)}(x)$ with $m=2,3, \ldots$. Let

$$
\mathbf{h}^{(m)}(x):=U^{(m)}(x)-U^{(m-1)}(x)
$$

then we have

$$
U^{(m)}(x)=U^{(0)}(x)+\mathbf{h}^{(1)}(x)+\sum_{i=2}^{m} \mathbf{h}^{(i)}(x) .
$$

We linearize nonlinear equations (2.3) around $U^{(m-1)}(x)$ to get the following boundary value problem

$$
\left\{\begin{array}{l}
\mathcal{L}\left[U^{m-1}\right]\left(\mathbf{h}^{(m)}\right)=\Pi_{N_{m}} E^{(m-1)}, \\
\nabla \cdot \mathbf{h}^{(m)}=0,
\end{array}\right.
$$

with the boundary conditions

$$
\left.\mathbf{h}^{(m)}(x)\right|_{x \in \partial \Omega}=0
$$

where the error term is given by

$$
\left.E^{(m-1)}:=\mathcal{J} U^{m-1}(x)\right]=\mathcal{R}\left(\mathbf{h}^{(m)}(x)\right),
$$

and 


$$
\begin{aligned}
\mathcal{R}\left(\mathbf{h}^{(m)}\right) & :=\mathcal{J}\left(U^{(m-1)}+\mathbf{h}^{(m)}\right)-\mathcal{J}\left(U^{(m-1)}\right)-\mathcal{L}\left[U^{(m-1)}\right]\left(\mathbf{h}^{(m)}\right) \\
& =\Pi_{N_{m}}\left(\mathbf{h}^{(m)} \cdot \nabla \mathbf{h}^{(m)}+\nabla P^{(m)}\right) .
\end{aligned}
$$

where

$$
P^{(m)}=-\Delta^{-1} \sum_{i, j=1}^{n} \frac{\partial h_{i}^{(m)}}{\partial x_{j}} \frac{\partial h_{j}^{(m)}}{\partial x_{i}} .
$$

This is also the nonlinear term in approximation problem (2.3) at $U^{(m-1)}(x)$.

The following result establishes how to construct the $m$-th approximation solution.

Proposition 3.1 Let $v \geq 1$. Assume the initial approximation function $U^{(0)}$ satisfying (2.4)-(2.6), $U^{(m-1)}(x) \in \mathcal{B}_{\varepsilon}$ and $\sum_{i=1}^{m-1}\left\|\mathbf{h}^{(i)}\right\|_{H^{s}}^{2} \lesssim \varepsilon^{2}$. Then the linearized problem (3.2) with the boundary condition (3.3) admits a Sobolev regular solution $\mathbf{h}^{(m)}(x) \in H^{s}(\Omega)$, which satisfies

$$
\left\|\mathbf{h}^{(m)}\right\|_{H^{s}}^{2} \lesssim\left\|\Pi_{N_{m}} E^{(m-1)}\right\|_{H^{s}}^{2}, \quad \forall t>0,
$$

where the error term satisfies

$$
\left\|E^{(m)}\right\|_{H^{s}}=\left\|\mathcal{R}\left(\mathbf{h}^{(m)}\right)\right\|_{H^{s}} \lesssim \varepsilon N_{m}^{2}\left\|\mathbf{h}^{(m)}\right\|_{H^{s}}^{2} .
$$

Proof Direct computation gives that

$$
\partial_{x_{i}} U_{j}^{(m-1)}(x)=\partial_{x_{i}} U_{j}^{(0)}(x)+\partial_{x_{i}} \mathbf{h}^{(1)}(x)+\sum_{i=2}^{m-1} \partial_{x_{i}} \mathbf{h}^{(i)}(x) .
$$

By the assumption $\sum_{i=1}^{m-1}\left\|\mathbf{h}^{(i)}\right\|_{H^{s}}^{2} \lesssim \varepsilon^{2}$, we observe that

$$
\partial_{x_{i}} U_{j}^{(m-1)}(x) \sim \partial_{x_{i}} U_{j}^{(0)}(x)+\mathcal{O}\left(\varepsilon^{2}\right) .
$$

Thus, noticing that $U^{(0)}(x)$ satisfies (2.4)-(2.6), by small modification of $\partial_{x_{i}} U_{j}^{(0)}(x)$, it follows that

$$
\sum_{k=0}^{s}\left\|\Pi_{N_{m}} \partial_{x_{i}}^{k} U_{j}^{(m-1)}(x)\right\|_{\mathbb{L}^{\infty}} \lesssim \varepsilon_{0}, \quad \forall i, j=1,2, \ldots, n .
$$

Moreover, we notice that the $(m-1)$-th approximation solution is

$$
U^{(m-1)}(x)=U^{(0)}(x)+\mathbf{h}^{(1)}(x)+\sum_{i=2}^{m-1} \mathbf{h}^{(i)}(x),
$$

and

$$
\nabla \cdot \mathbf{h}^{(m-1)}=0
$$

Thus, it follows that 


$$
\left\{\begin{array}{l}
\nabla \cdot U^{(m-1)}(x)=0, \\
\left\|U^{(m-1)}\right\|_{H^{s}} \lesssim \varepsilon, \\
\left.U^{(m-1)}(x)\right|_{x \in \partial \Omega}=0 .
\end{array}\right.
$$

Then we will find the $m$-th $(m \geq 2)$ approximation solution $U^{(m)}(x)$, which is equivalent to find $\mathbf{h}^{(m)}(x)$ such that

$$
U^{(m)}(x)=U^{(m-1)}(x)+\mathbf{h}^{(m)}(x) .
$$

Substituting (3.10) into (2.3), it follows that

$$
\mathcal{J}\left(U^{(m)}\right)=\mathcal{J}\left(U^{(m-1)}\right)+\mathcal{L}\left[U^{(m-1)}\right] \mathbf{h}^{(m)}+\mathcal{R}\left(\mathbf{h}^{(m)}\right) .
$$

Setting

$$
\mathcal{L}\left[U^{(m-1)}\right] \mathbf{h}^{(m)}=-\mathcal{J}\left(U^{(m-1)}\right)=-E^{(m-1)},
$$

we supplement it with the boundary conditions (3.3).

Since we assume $U^{(m-1)}(x) \in \mathcal{B}_{\varepsilon}$, there is the same structure between the linear system (2.9) and the linear system of $m$ th approximation solutions. Thus, by means of the same proof process in Proposition 2.1, we can show that the above problem admits a solution $\mathbf{h}^{(m)}(x) \in H^{s}(\Omega)$. For this purpose, we should use (2.2). Furthermore, similar to (2.50), we can use (3.8)-(3.9) to derive

$$
\left\|\mathbf{h}^{(m)}\right\|_{H^{s}}^{2} \lesssim\left\|E^{(m-1)}\right\|_{H^{s}}^{2}, \quad \forall t>0,
$$

where one can see the $(m-1)$-th error term $E^{(m-1)}$ such that

$$
E^{(m-1)}:=\mathcal{J}\left(U^{(m-1)}\right)=\mathcal{R}\left(\mathbf{h}^{(m)}\right) .
$$

Moreover, by (3.5) and the standard Calderon-Zygmund theory, it follows that

$$
\left\|E^{(m)}\right\|_{H^{s}}=\left\|\Pi_{N_{m}}\left(\mathbf{h}^{(m)} \cdot \nabla \mathbf{h}^{(m)}+\nabla P^{(m)}\right)\right\|_{H^{s}} \lesssim N_{m}^{2}\left\|\mathbf{h}^{(m)}\right\|_{H^{s}}^{2} .
$$

The proof is now complete.

\section{Convergence of the approximation scheme}

Our target is to prove that $U^{(\infty)}(x)$ is a global solution of nonlinear equations (1.1). This is equivalent to show that the series $\sum_{i=1}^{m} \mathbf{h}^{(i)}(x)$ is convergent.

For a fixed constant $s \geq 1$, let $1 \leq s=\bar{k}<k_{0} \leq k$ and

$$
\begin{aligned}
& k_{m}:=\bar{k}+\frac{k-\bar{k}}{2^{m}}, \quad k_{+\infty}=\bar{k}, \\
& \alpha_{m+1}:=k_{m}-k_{m+1}=\frac{k-\bar{k}}{2^{m+1}},
\end{aligned}
$$

which gives that 


$$
k_{0}>k_{1}>\ldots>k_{m}>k_{m+1}>\ldots
$$

Proposition 4.1 Let $v \geq 1$. Assume the initial approximation function $U^{(0)}$, satisfying (2.4)-(2.6). Then the steady incompressible Navier-Stokes equations (1.1) with the Dirichlet boundary condition (1.2) admit a global Sobolev solution

$$
U^{(\infty)}(x)=U^{(0)}(x)+\sum_{m=1}^{\infty} \mathbf{h}^{(m)}(x) \in H^{s}(\Omega),
$$

Moreover, the following estimate holds

$$
\left\|U^{(\infty)}\right\|_{H^{s}} \lesssim \varepsilon
$$

Proof The proof is based on the induction. Note that $N_{m}=N_{0}^{m}$ with $N_{0}>1$. For all $m=1,2, \ldots$, we claim that there exists a sufficient small positive constant $\varepsilon$ such that

$$
\begin{aligned}
& \left\|\mathbf{h}^{(m)}\right\|_{H^{k_{m-1}}} \lesssim \varepsilon^{2^{m-1}}, \\
& \left\|E^{(m)}\right\|_{H^{k_{m-1}}} \lesssim \varepsilon^{2^{m}}, \\
& U^{(m)} \in \mathcal{B}_{\varepsilon} .
\end{aligned}
$$

For the case of $m=1$, we recall the assumptions (2.4)-(2.6) on the initial approximation function $U^{(0)}(x)$. By (2.50), taking $0<\varepsilon<N_{0}^{-(8+k-k)} \varepsilon^{2} \ll 1$, it follows that

$$
\left\|\mathbf{h}^{(1)}\right\|_{H^{k_{0}}} \lesssim\left\|E^{(0)}\right\|_{H^{k_{0}}}<\varepsilon_{0}<\varepsilon^{2} .
$$

Moreover, by (3.7) and the above estimate, we have

$$
\left\|E^{(1)}\right\|_{H^{k_{0}}} \lesssim\left\|\mathcal{R}\left(\mathbf{h}^{1)}\right)\right\|_{H^{k_{0}}} \lesssim N_{1}^{2}\left\|\mathbf{h}^{(1)}\right\|_{H^{k_{0}}}^{2} \lesssim \varepsilon^{2},
$$

and

$$
\left\|U^{(1)}\right\|_{H^{k_{0}}} \lesssim\left\|U^{(0)}\right\|_{H^{k_{0}}}+\left\|\mathbf{h}^{(1)}\right\|_{H^{k_{0}}} \lesssim \varepsilon,
$$

which means that $U^{(1)} \in \mathcal{B}_{\varepsilon}$.

Assume that the case of $(m-1)$ holds, that is,

$$
\begin{aligned}
& \left\|\mathbf{h}^{(m-1)}\right\|_{H^{k_{m}}}<\varepsilon^{2^{m-2}}, \\
& \left\|E^{(m-1)}\right\|_{H^{k_{m}}}<\varepsilon^{2^{m-1}}, \\
& U^{(m-1)} \in \mathcal{B}_{\varepsilon} .
\end{aligned}
$$

We now prove that the case of $m$ holds. Using (2.2), (3.6) and the second inequality of (4.3), we obtain

$$
\begin{aligned}
\left\|\mathbf{h}^{(m)}\right\|_{H^{k_{m-1}}} & \lesssim\left\|\Pi_{N_{m}} E^{(m-1)}\right\|_{H^{k_{m-1}}} \\
& \lesssim N_{m}^{\alpha_{m}}\left\|E^{(m-1)}\right\|_{H^{k_{m}}} \\
& <\varepsilon^{2^{m-2}} .
\end{aligned}
$$

Combining this estimate with (2.2), (3.7) and (4.1), we obtain 


$$
\begin{aligned}
\left\|E^{(m)}\right\|_{H^{k_{m}}} & \lesssim N_{m}^{2}\left\|\mathbf{h}^{(m)}\right\|_{H^{k_{m}}}^{2} \\
& \lesssim N_{m}^{2+\alpha_{m+1}}\left(\left\|E^{(m-1)}\right\|_{H^{k_{m+1}}}\right)^{2} \\
& \lesssim\left(N_{0}\right)^{\left(2+\alpha_{m+1}\right) m+2\left(2+\alpha_{m+2}\right)(m-1)}\left(\left\|E^{(m-2)}\right\|_{H^{k_{m+2}}}\right)^{2^{2}} \\
& \lesssim \ldots \\
& \lesssim\left(N_{0}^{8+k-\bar{k}}\left\|E^{(0)}\right\|_{H^{k_{2 m}}}\right)^{2^{m}} .
\end{aligned}
$$

We choose a sufficiently small positive constant $\varepsilon_{0}$ such that

$$
0<N_{0}^{8+k-\bar{k}}\left\|E^{(0)}\right\|_{H^{\bar{k}}}<N_{0}^{4} \varepsilon_{0} \lesssim \varepsilon^{2} .
$$

Thus, by (4.5) we have

$$
\left\|E^{(m)}\right\|_{H^{k_{m}}} \lesssim \varepsilon^{2^{m}}
$$

and

$$
0 \leq \lim _{m \rightarrow+\infty}\left\|E^{(m)}\right\|_{H^{k_{m}}} \lesssim\left(N_{0}^{8+k-\bar{k}}\left\|E^{(0)}\right\|_{H^{k_{+}}}\right)^{2^{+\infty}} \longrightarrow 0 .
$$

So, the error term goes to 0 as $m \rightarrow \infty$, that is,

$$
\lim _{m \rightarrow \infty}\left\|E^{(m)}\right\|_{H^{k_{m}}}=0 .
$$

On the other hand, note that $N_{m}=N_{0}^{m}$, by (4.3)-(4.4). It follows that

$$
\begin{aligned}
\left\|U^{(m)}\right\|_{H^{k_{m}}} & \lesssim\left\|U^{(m-1)}\right\|_{H^{k_{m}}}+\left\|\mathbf{h}^{(m)}\right\|_{H^{k_{m}}} \\
& \lesssim \varepsilon+N_{m}^{3} \varepsilon^{2^{m}} \lesssim \varepsilon .
\end{aligned}
$$

This means that $U^{(m)} \in \mathcal{B}_{\varepsilon}$. Hence we conclude that (4.2) holds.

Therefore, the steady incompressible Navier-Stokes equations (1.1) with the Dirichlet boundary condition admit global solutions

$$
U^{(\infty)}(x)=U^{(0)}(x)+\sum_{m=1}^{\infty} \mathbf{h}^{(m)}(x)=U^{(0)}(x)+\mathcal{O}\left(\varepsilon^{2}\right),
$$

from which, one can see the solution depends on the initial approximation function $U^{(0)}(x)$ strongly.

Finally, by (1.3) and the standard Calderon-Zygmund theory (that is, for the Riesz operator $\mathcal{R}$ ), we have $\|\mathcal{R} U\|_{\mathbb{L}^{s_{0}}} \leq\|U\|_{\mathbb{L}^{s_{0}}}$ with $1<s_{0}<\infty$. We conclude that

$$
\|P\|_{H^{s}} \lesssim \varepsilon
$$

and this completes the proof.

Acknowledgements W. Yan is supported by the National Natural Science Foundation of China (No. 11771359). V.D. Rădulescu acknowledges the support of the Slovenian Research Agency Grants P1-0292, J1-8131, J1-7025, N1-0064, and N1-0083. 
Open Access This article is licensed under a Creative Commons Attribution 4.0 International License, which permits use, sharing, adaptation, distribution and reproduction in any medium or format, as long as you give appropriate credit to the original author(s) and the source, provide a link to the Creative Commons licence, and indicate if changes were made. The images or other third party material in this article are included in the article's Creative Commons licence, unless indicated otherwise in a credit line to the material. If material is not included in the article's Creative Commons licence and your intended use is not permitted by statutory regulation or exceeds the permitted use, you will need to obtain permission directly from the copyright holder. To view a copy of this licence, visit http://creativecommons.org/licenses/by/4.0/.

\section{References}

1. Alinhac, S.: Existence d'ondes de raréfaction pour des systèmes quasi-linéaires hyperboliques multidimensionnels. Commun. Partial Differ. Equ. 14(2), 173-230 (1989)

2. Al Baba, H.: Maximal $L^{p}-L^{q}$ regularity to the Stokes problem with Navier boundary conditions. Adv. Nonlinear Anal. 8, 743-761 (2019)

3. Beirao da Veiga, H., Yang, J.Q.: Regularity criteria for Navier-Stokes equations with slip boundary conditions on non-flat boundaries via two velocity components. Adv. Nonlinear Anal. 9, 633-643 (2020)

4. Brandolese, L.: Fine properties of self-similar solutions of the Navier-Stokes equations. Arch. Ration. Mech. Anal. 192, 375-401 (2009)

5. Buckmaster, T., Vicol, V.: Nonuniqueness of weak solutions to the Navier-Stokes equation. Ann. Math. 189, 101-144 (2019)

6. Caffarelli, L., Kohn, R.V., Nirenberg, L.: Partial regularity of suitable weak solutions of the Navier-Stokes equations. Commun. Pure Appl. Math. 35, 771-831 (1982)

7. Farwig, R., Sohr, H.: Existence, uniqueness and regularity of stationary solutions to inhomogeneous Navier-Stokes equations in $\mathbb{R}^{n}$. Czechoslov. Math. J. 59, 61-79 (2009)

8. Fefferman, C.L.: Existence and smoothness of the Navier-Stokes equations. Millenn. Prize Probl. 57, 67 (2006)

9. Foiaş, C., Manley, O., Rosa, R., Temam, R.: Navier-Stokes Equations and Turbulence. Encyclopedia of Mathematics and Its Applications, vol. 83. Cambridge University Press, Cambridge (2001)

10. Frehse, J., Ruzicka, M.: On the regularity of the stationary Navier-Stokes equations. Ann. Sc. Norm. Super. Pisa Cl. Sci. 21, 63-95 (1994)

11. Frehse, J., Ruzicka, M.: Existence of regular solutions to the stationary Navier-Stokes equations. Math. Ann. 302, 699-717 (1995)

12. Frehse, J., Ruzicka, M.: Regular solutions to the steady Navier-Stokes equations. In: Sequeira, A. (ed.) Navier-Stokes Equations and Related Nonlinear Problems (Funchal, 1994), pp. 131-139. Plenum, New York (1995)

13. Galdi, G.P.: An Introduction to the Mathematical Theory of the Navier-Stokes Equations II. Nonlinear Steady Problems. Springer Tracts in Natural Philosophy, vol. 39. Springer, New York (1994)

14. Galdi, G.P., Simader, C.G., Sohr, $\mathrm{H}_{\mathrm{r}}$ : A class of solutions to stationary stokes and Navier-Stokes equations with boundary data in $W^{-\frac{1}{q}, q}$. Math. Ann. 331, 41-74 (2005)

15. Gerhardt, C.: Stationary solutions to the Navier-Stokes equations in dimension four. Math. Z. 165, 193-197 (1979)

16. Gilbarg, D., Trudinger, N.S.: Elliptic Partial Differential Equations of Second Order. Grundlehren der Mathematischen Wissenschaften, vol. 224, 2nd edn. Springer, Berlin (1983)

17. Hörmander, L.: Implicit Function Theorems. Stanford Lecture Notes, University of Stanford (1977)

18. Hou, Y., Pei, S.: On the weak solutions to steady Navier-Stokes equations with mixed boundary conditions. Math. Z. 291, 47-54 (2019)

19. Jia, H., Šverák, V.: Local in space estimates near initial time for weak solutions of the NavierStokes equations and forward self-similar solutions. Invent. Math. 196, 233-265 (2014)

20. Kim, H.: Existence and regularity of very weak solutions of the stationary Navier-Stokes equations. Arch. Ration. Mech. Anal. 193, 117-152 (2009)

21. Koch, H., Tataru, D.: Well-posedness for the Navier-Stokes equations. Adv. Math. 157, 22-35 (2001)

22. Leray, J.: Sur le mouvement d'un liquide visqueux emplissant l'espace. Acta Math. 63, 193-248 (1934)

23. Lin, F.: A new proof of the Caffarelli-Kohn-Nirenberg theorem. Commun. Pure Appl. Math. 51, 241257 (1998) 
24. Luo, X.: Stationary solutions and nonuniqueness of weak solutions for the Navier-Stokes equations in high dimensions. Arch. Ration. Mech. Anal. 233, 701-747 (2019)

25. Maz'ya, V., Rossmann, J.: Mixed boundary value problems for the stationary Navier-Stokes system in polyhedral domains. Arch. Ration. Mech. Anal. 194, 669-712 (2009)

26. Moser, J.: A rapidly converging iteration method and nonlinear partial differential equations I-II. Ann. Scuola Norm. Sup. Pisa. 20, 265-313 (1966)

27. Nash, J.: The embedding for Riemannian manifolds. Am. J. Math. 63, 20-63 (1956)

28. Necas, J., Ruzicka, M., Śverák, V.: On Leray's self-similar solutions of the Navier-Stokes equations. Acta Math. 176, 283-294 (1996)

29. Rabinowitz, P.H.: A rapid convergence method for a singular perturbation problem. Ann. Inst. H. Poincaré, Anal. Non Linéaire 1, 1-17 (1984)

30. Safonov, M.V.: Nonlinear Elliptic Equations of the Second Order. Lecture notes, University of Florence, Italy (1991)

31. Struwe, M.: Regular solutions of the stationary Navier-Stokes equations on $\mathbb{R}^{5}$. Math. Ann. 302, 719741 (1995)

32. Temam, R.: Navier-Stokes Equations and Nonlinear Functional Analysis. In: CBMS-NSF Regional Conference Series in Applied Mathematics, vol. 66, Society for Industrial and Applied Mathematics (SIAM), Philadelphia, PA (1995)

33. Temam, R.: Navier-Stokes Equations, Theory and Numerical Analysis. AMS Chelsea Publishing, Providence (2001)

34. Wang, Y.Z., Jie, X.: Well/ill-posedness for the dissipative Navier-Stokes system in generalized Carleson measure spaces. Adv. Nonlinear Anal. 8, 203-224 (2019)

35. Yan, W.P.: The motion of closed hypersurfaces in the central force field. J. Differ. Equ. 261, 19732005 (2016)

36. Yan, W.P.: Dynamical behavior near explicit self-similar blow up solutions for the Born-Infeld equation. Nonlinearity 32, 4682-4712 (2019)

37. Yan, W.P., Zhang, B.L.: Long time existence of solution for the bosonic membrane in the light cone gauge. J. Geom. Anal. (2019). https://doi.org/10.1007/s12220-019-00269-1

38. Zhao, X., Yan, W.P.: Existence of standing waves for quasi-linear Schrödinger equations on $\mathbb{T}^{n}$. Adv. Nonlinear Anal. 9, 978-993 (2020)

Publisher's Note Springer Nature remains neutral with regard to jurisdictional claims in published maps and institutional affiliations. 